\title{
PRINCIPAL COMPONENT ANALYSIS FOR SECOND-ORDER STATIONARY VECTOR TIME SERIES
}

\author{
By JinYUAN ChANG ${ }^{*, 1}$, Bin GUO ${ }^{*, 2}$ AND QIWEI YAO ${ }^{\dagger, \ddagger, 3}$ \\ Southwestern University of Finance and Economics*, \\ London School of Economics and Political Science $e^{\dagger}$ and \\ Guanghua School of Management, Peking University
}

We extend the principal component analysis (PCA) to second-order stationary vector time series in the sense that we seek for a contemporaneous linear transformation for a $p$-variate time series such that the transformed series is segmented into several lower-dimensional subseries, and those subseries are uncorrelated with each other both contemporaneously and serially. Therefore, those lower-dimensional series can be analyzed separately as far as the linear dynamic structure is concerned. Technically, it boils down to an eigenanalysis for a positive definite matrix. When $p$ is large, an additional step is required to perform a permutation in terms of either maximum crosscorrelations or FDR based on multiple tests. The asymptotic theory is established for both fixed $p$ and diverging $p$ when the sample size $n$ tends to infinity. Numerical experiments with both simulated and real data sets indicate that the proposed method is an effective initial step in analyzing multiple time series data, which leads to substantial dimension reduction in modelling and forecasting high-dimensional linear dynamical structures. Unlike PCA for independent data, there is no guarantee that the required linear transformation exists. When it does not, the proposed method provides an approximate segmentation which leads to the advantages in, for example, forecasting for future values. The method can also be adapted to segment multiple volatility processes.

1. Introduction. Modelling multiple time series, also called vector time series, is always a challenge, even when the vector dimension $p$ is moderately large. While most inference methods and the associated theory for univariate autoregressive and moving average (ARMA) processes have found their multivariate counterparts [Lütkepohl (2005)], vector autoregressive and moving average (VARMA)

\footnotetext{
Received September 2016; revised July 2017.

${ }^{1}$ Supported in part by the Fundamental Research Funds for the Central Universities (Grant No. JBK171121, JBK170161, JBK150501), NSFC (Grant No. 11501462), the Center of Statistical Research at SWUFE, and the Joint Lab of Data Science and Business Intelligence at SWUFE.

${ }^{2}$ Supported in part by the Fundamental Research Funds for the Central Universities (Grant No. JBK120509, JBK140507), NSFC (Grant No. 11601356), China's National Key Research Special Program Grant 2016YFC0207702 and the Center of Statistical Research at SWUFE.

${ }^{3}$ Supported in part by an EPSRC research grant.

MSC2010 subject classifications. Primary 62M10; secondary 62H25.
}

Key words and phrases. $\alpha$-mixing, autocorrelation, cross-correlation, dimension reduction, eigenanalysis, high-dimensional time series, weak stationarity. 
models are seldom used directly in practice when $p \geq 3$. This is partially due to the lack of identifiability for VARMA models in general. More fundamentally, those models are overparametrized, leading to flat likelihood functions which cause innate difficulties in statistical inference. Therefore, finding an effective way to reduce the number of parameters is particularly felicitous in modelling and forecasting multiple time series. The urge for doing so is more pertinent in this modern information age, as it has become commonplace to access and to analyze highdimensional time series data with dimension $p$ in the order of hundreds or more. Big time series data arise from, among others, panel study for economic and natural phenomena, social network, healthcare and public health, financial market, supermarket transactions, information retrieval and recommender systems.

Available methods to reduce the number of parameters in modelling vector time series can be divided into two categories: regularization and dimension reduction. The former imposes some conditions on the structure of a VARMA model. The latter represents a high-dimensional process in terms of several lower-dimensional processes. Various regularization methods have been developed in literature. For example, Jakeman, Steele and Young (1980) adopted a two-stage regression strategy based on instrumental variables to avoid using moving average explicitly. Different canonical structures are imposed on VARMA models [Chapter 3 of Reinsel (1993), Chapter 4 of Tsay (2014), and references within]. Structural restrictions are imposed in order to specify and to estimate some reduced forms of vector autoregressive (VAR) models [Chapter 9 of Lütkepohl (2005), and references within]. Davis, Zang and Zheng (2016) proposed a VAR model with sparse coefficient matrices based on partial spectral coherence. Under different sparsity assumptions, VAR models have been estimated by LASSO regularization [Shojaie and Michailidis (2010), Song and Bickel (2011)], or by the Dantzig selector [Han, Lu and Liu (2015)]. Guo, Wang and Yao (2016) considered high-dimensional autoregression with banded coefficient matrices. The dimension reduction methods include the canonical correlation analysis of Box and Tiao (1977), the independent component analysis (ICA) of Back and Weigend (1997), the principal component analysis (PCA) of Stock and Watson (2002), the scalar component analysis of Tiao and Tsay (1989) and Huang and Tsay (2014), the dynamic orthogonal components analysis of Matteson and Tsay (2011). Another popular approach is to represent multiple time series in terms of a few latent factors defined in various ways. There is a large body of literature in this area published in the outlets in statistics, econometrics and signal processing. An incomplete list of the publications includes Anderson (1963), Peña and Box (1987), Bai and Ng (2002), Theis, Meyer-Baese and Lang (2004), Stock and Watson (2005), Forni et al. (2005), Pan and Yao (2008), Lam, Yao and Bathia (2011), Lam and Yao (2012) and Chang, Guo and Yao (2015).

A new dimension reduction method is proposed in this paper. We seek for a contemporaneous linear transformation such that the transformed series is segmented into several lower-dimensional subseries, and those subseries are uncorrelated with 
each other both contemporaneously and serially. Therefore, they can be modelled or forecasted separately, as far as linear dependence is concerned. This reduces the number of parameters involved in depicting linear dynamic structure substantially. While the basic idea is not new, which has been explored with various methods including some aforementioned references, the method proposed in this paper (i.e., the new PCA for time series) is new, simple and effective. Technically, the proposed method boils down to an eigenanalysis for a positive definite matrix, which is a quadratic function of the cross correlation matrix function for the observed process. Hence it is easy to implement and the required computation can be carried out with, for example, an ordinary personal computer or laptop for the data with dimension $p$ in the order of thousands.

The method can be viewed as an extension of the standard PCA for multiple time series, therefore, is abbreviated as TS-PCA. However the segmented subseries are not guaranteed to exist as those subseries must not correlate with each other across all times. This is a marked difference from the standard PCA. The real data examples in Section 4 indicate that it is often reasonable to assume that the segmentation exists. Furthermore, when the assumption is invalid, the proposed method provides some approximate segmentations which ignore some weak though significant correlations, and those weak correlations are of little practical use for modelling and forecasting. Thus the proposed method can be used as an initial step in analyzing multiple time series, which often transforms a multidimensional problem into several lower-dimensional problems. Chang, Yao and Zhou (2017) demonstrates that such an initial transformation increases the power in testing for high-dimensional white noise. Furthermore, the results obtained for the transformed subseries can be easily transformed back to the original multiple time series. Illustration with real data examples indicates clearly the advantages in post-sample forecasting from using the proposed TS-PCA. The $R$-package PCA4TS, available from CRAN project, implements the proposed methodology.

The proposed TS-PCA can be viewed as a version of ICA. In fact, our goal is the same in principle as the ICA using autocovariances presented in Section 18.1 of Hyvärinen, Karhunen and Oja (2001). However, the nonlinear optimization algorithms presented there are to search for a linear transformation such that all the off-diagonal elements of the autocovariance matrices for the transformed vector time series are minimized; see also Tong, Xu and Kailath (1994) and Belouchrani et al. (1997). To apply those algorithms to our setting, we need to know exactly the block diagonal structure of autocovariances of the transformed vector process (i.e., the number of blocks and the sizes of all the blocks), which is unknown in practice. Furthermore, our method is simple and fast and, therefore, is applicable to highdimensional cases. Cardoso (1998) extends the basic idea of ICA to the so-called multivariate ICA, which requires the transformed random vector to be segmented into several independent groups with possibly more than one component in each 
group. But Cardoso (1998) does not provide a pertinent algorithm for multivariate ICA. Furthermore, it does not consider the dependence across different time lags. TS-PCA is also different from the dynamic PCA proposed in Chapter 9 of Brillinger (1975), which decomposes each component time series as the sum of moving averages of several uncorrelated white noise processes. In our TS-PCA, no lagged variables enter the decomposition.

The rest of the paper is organized as follows. The methodology is spelled out in Section 2. Section 3 presents the associated asymptotic properties of the proposed method. Numerical illustration with real data are reported in Section 4. Section 5 extends the method to segmenting a multiple volatility process into several lowerdimensional volatility processes. Some final remarks are given in Section 6. All technical proofs and numerical illustration with simulated data are relegated to the supplementary material [Chang, Guo and Yao (2018)]. We always use the following notation. For any $m \times k$ matrix $\mathbf{H}=\left(h_{i, j}\right)_{m \times k}$, let $\|\mathbf{H}\|_{2}=\lambda_{\max }^{1 / 2}\left(\mathbf{H H}^{\mathrm{T}}\right)$ and $\|\mathbf{H}\|_{F}=\left(\sum_{i=1}^{m} \sum_{j=1}^{k} h_{i, j}^{2}\right)^{1 / 2}$, where $\lambda_{\max }\left(\mathbf{H H}^{\mathrm{T}}\right)$ denotes the largest eigenvalue of $\mathbf{H H}^{\mathrm{T}}$.

\section{Methodology.}

2.1. Setting and method. Let $\mathbf{y}_{t}$ be observable $p \times 1$ weakly stationary time series. We assume that $\mathbf{y}_{t}$ admits a latent segmentation structure:

$$
\mathbf{y}_{t}=\mathbf{A} \mathbf{x}_{t},
$$

where $\mathbf{x}_{t}$ is an unobservable $p \times 1$ weakly stationary time series consisting of $q(>1)$ both contemporaneously and serially uncorrelated subseries, and $\mathbf{A}$ is an unknown constant matrix. Hence all the autocovariances of $\mathbf{x}_{t}$ are of the same block-diagonal structure with $q$ blocks. Denote the segmentation of $\mathbf{x}_{t}$ by

$$
\mathbf{x}_{t}=\left\{\left(\mathbf{x}_{t}^{(1)}\right)^{\mathrm{T}}, \ldots,\left(\mathbf{x}_{t}^{(q)}\right)^{\mathrm{T}}\right\}^{\mathrm{T}}
$$

with $\operatorname{Cov}\left\{\mathbf{x}_{t}^{(i)}, \mathbf{x}_{s}^{(j)}\right\}=\mathbf{0}$ for all $t, s$ and $i \neq j$. Therefore, $\mathbf{x}_{t}^{(1)}, \ldots, \mathbf{x}_{t}^{(q)}$ can be modelled or forecasted separately as far as their linear dynamic structure is concerned.

EXAMPLE 1. Before we spell out how to find the segmentation transformation $\mathbf{A}$ in general, we consider the monthly temperatures of 7 cities (Nanjing, Dongtai, Huoshan, Hefei, Shanghai, Anqing and Hangzhou) in Eastern China from January 1954 to December 1998. Figure 1(a) plots the cross correlations of these 7 temperature time series. Both the autocorrelation of each component series and the cross correlation between any two component series are dominated by the annual temperature fluctuation; showing the strong periodicity with the period 12 . Now 


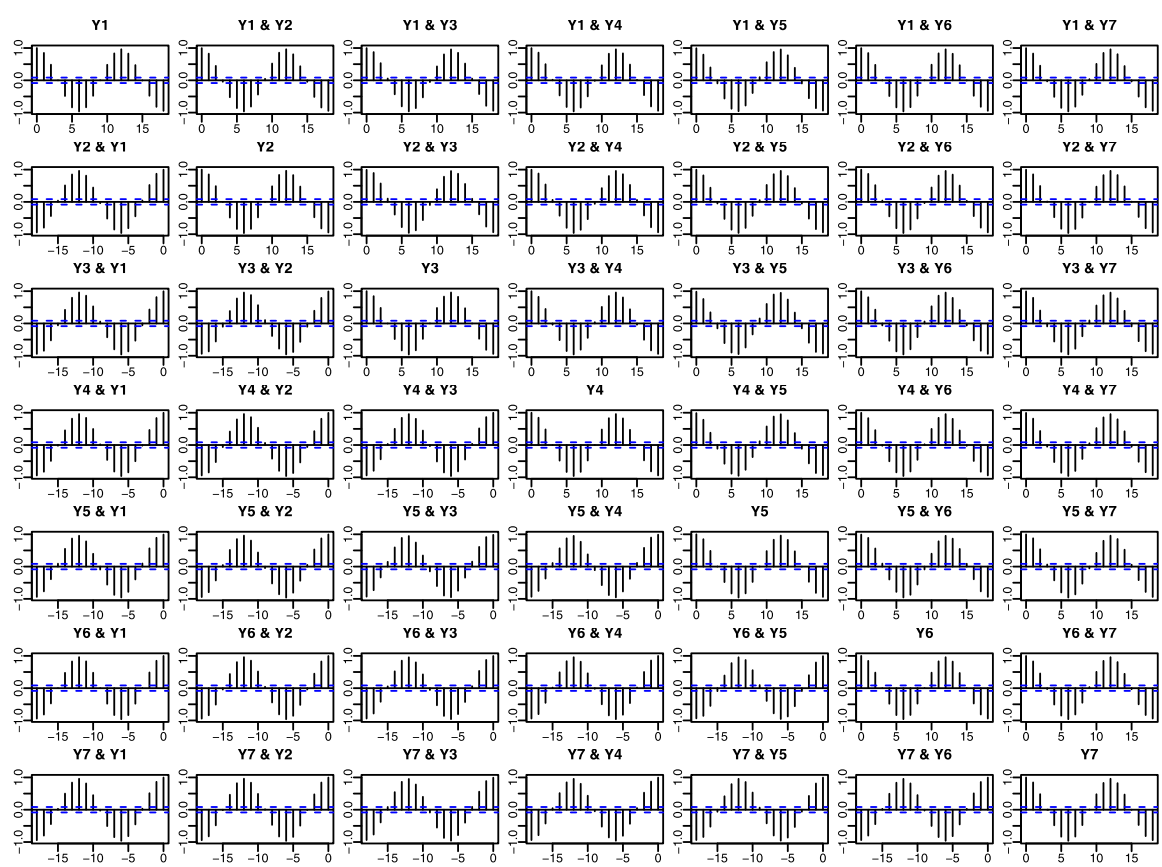

(a) Cross correlogram of the 7 original temperature time series

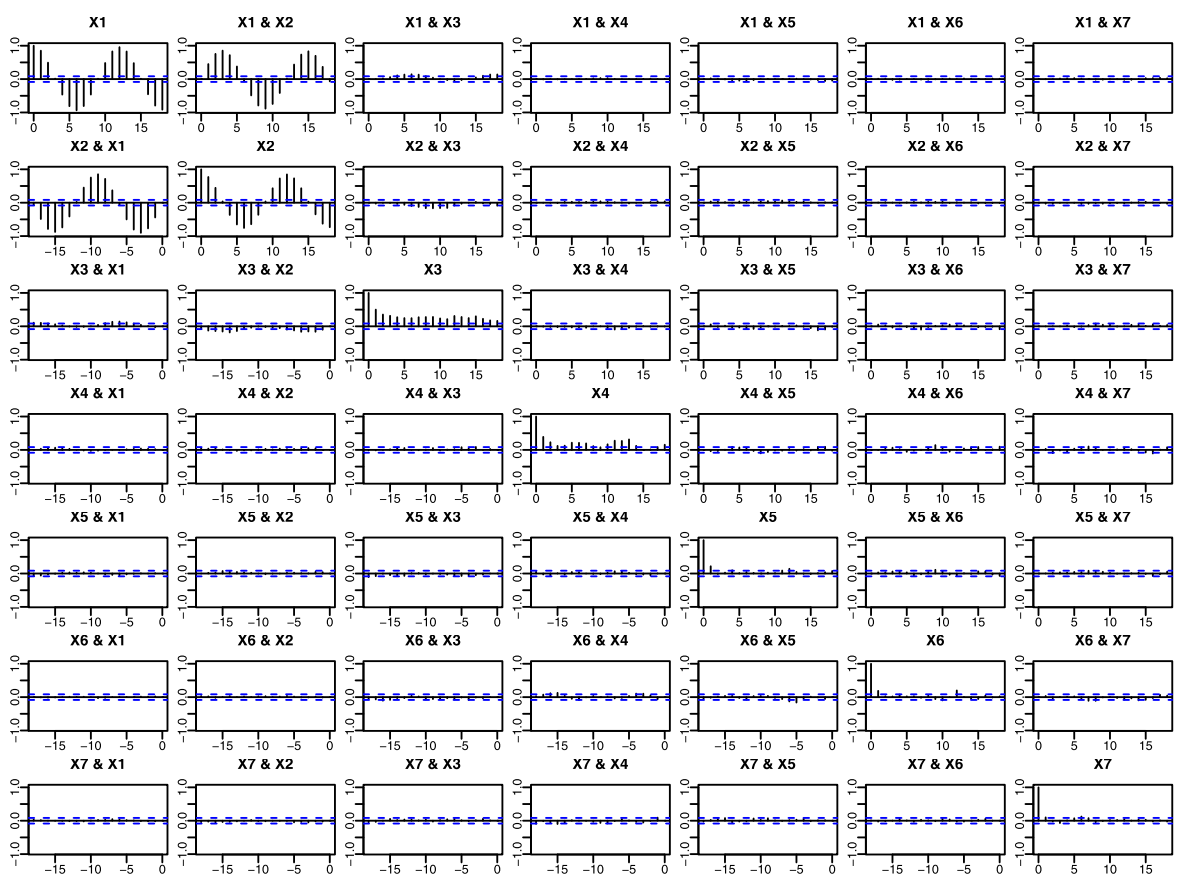

(b) Cross correlogram of the 7 transformed time series

FIG. 1. Cross correlograms for Example 1. 
we apply the linear transformation $\mathbf{x}_{t}=\mathbf{B} \mathbf{y}_{t}$ with

$$
\mathbf{B}=\left(\begin{array}{ccccccc}
0.244 & -0.066 & 0.019 & -0.050 & -0.313 & -0.154 & 0.200 \\
-0.703 & 0.324 & -0.617 & 0.189 & 0.633 & 0.499 & -0.323 \\
0.375 & 1.544 & -1.615 & 0.170 & -2.266 & 0.126 & 1.596 \\
3.025 & -1.381 & -0.787 & -1.691 & -0.212 & 1.188 & -0.165 \\
-0.197 & -1.820 & -1.416 & 3.269 & 0.301 & -1.438 & 1.299 \\
-0.584 & -0.354 & 0.847 & -1.262 & -0.218 & -0.151 & 1.831 \\
1.869 & -0.742 & 0.034 & 0.501 & 0.492 & -2.533 & 0.339
\end{array}\right) .
$$

See Section 4 for how $\mathbf{B}$ is calculated. Figure 1(b) shows that the first two transformed component series are significantly correlated both concurrently and serially, and there are also small but significant correlations in the $(3,2)$ th panel; indicating the linear dependence between the 2 nd and the 3 rd transformed component series. Apart from these, there is little significant cross correlation among all the other pairs of component series. This visual observation suggests to segment the 7 transformed series into 5 uncorrelated groups: $\{1,2,3\},\{4\},\{5\},\{6\}$ and $\{7\}$.

This example indicates that the segmentation transformation transfers the problem of analyzing a 7-dimensional time series into the five lower-dimensional problems: four univariate time series and one 3-dimensional time series. Those five time series can and should be analyzed separately as there are no cross correlations among them at all time lags. The linear dynamic structure of the original series is deduced by those of the five transformed series, as $\operatorname{Cov}\left(\mathbf{y}_{t+k}, \mathbf{y}_{t}\right)=$ $\mathbf{A} \operatorname{Cov}\left(\mathbf{x}_{t+k}, \mathbf{x}_{t}\right) \mathbf{A}^{\mathrm{T}}$.

Now we spell out how to find the segmentation transformation under (2.1) and (2.2). Without the loss of generality, we may assume

$$
\operatorname{Var}\left(\mathbf{y}_{t}\right)=\mathbf{I}_{p} \text { and } \operatorname{Var}\left(\mathbf{x}_{t}\right)=\mathbf{I}_{p},
$$

where $\mathbf{I}_{p}$ denotes the $p \times p$ identity matrix. This first equation in (2.3) amounts to replace $\mathbf{y}_{t}$ by $\widehat{\mathbf{V}}^{-1 / 2} \mathbf{y}_{t}$ as a preliminary step in practice, where $\widehat{\mathbf{V}}$ is a consistent estimator for $\operatorname{Var}\left(\mathbf{y}_{t}\right)$. As both $\mathbf{A}$ and $\mathbf{x}_{t}$ are unobservable, the second equation in (2.3) implies that we view $\left(\mathbf{A}\left\{\operatorname{Var}\left(\mathbf{x}_{t}\right)\right\}^{1 / 2},\left\{\operatorname{Var}\left(\mathbf{x}_{t}\right)\right\}^{-1 / 2} \mathbf{x}_{t}\right)$ as $\left(\mathbf{A}, \mathbf{x}_{t}\right)$ in (2.1). More importantly, the latter perspective will not alter the block-diagonal structure of the autocovariance matrices of $\mathbf{x}_{t}$. Now it follows from (2.1) and (2.3) that $\mathbf{I}_{p}=$ $\operatorname{Var}\left(\mathbf{y}_{t}\right)=\mathbf{A} \operatorname{Var}\left(\mathbf{x}_{t}\right) \mathbf{A}^{\mathrm{T}}=\mathbf{A} \mathbf{A}^{\mathrm{T}}$. Thus, $\mathbf{A}$ in (2.1) is an orthogonal matrix under (2.3).

Let $p_{j}$ be the length of $\mathbf{x}_{t}^{(j)}$. Write $\mathbf{A}=\left(\mathbf{A}_{1}, \ldots, \mathbf{A}_{q}\right)$, where $\mathbf{A}_{j}$ has $p_{j}$ columns. Since $\mathbf{x}_{t}=\mathbf{A}^{\mathrm{T}} \mathbf{y}_{t}$, it follows from (2.2) that

$$
\mathbf{x}_{t}^{(j)}=\mathbf{A}_{j}^{\mathrm{T}} \mathbf{y}_{t}, \quad j=1, \ldots, q .
$$

Let $\mathbf{H}_{j}$ be any $p_{j} \times p_{j}$ orthogonal matrix, and $\mathbf{H}=\operatorname{diag}\left(\mathbf{H}_{1}, \ldots, \mathbf{H}_{q}\right)$. Then $\left(\mathbf{A}, \mathbf{x}_{t}\right)$ in (2.1) can be replaced by $\left(\mathbf{A H}, \mathbf{H}^{\mathrm{T}} \mathbf{x}_{t}\right)$ while the block structure as in 
(2.2) still holds. Hence $\mathbf{A}$ and $\mathbf{x}_{t}$ are not uniquely identified in (2.1), even with the additional assumption (2.3). In fact, under (2.3), only $\mathcal{M}\left(\mathbf{A}_{1}\right), \ldots, \mathcal{M}\left(\mathbf{A}_{q}\right)$ are uniquely defined by (2.1), where $\mathcal{M}\left(\mathbf{A}_{j}\right)$ denotes the linear space spanned by the columns of $\mathbf{A}_{j}$. Consequently, $\boldsymbol{\Gamma}_{j}^{\mathrm{T}} \mathbf{y}_{t}$ can be taken as $\mathbf{x}_{t}^{(j)}$ for any $p \times p_{j}$ matrix $\boldsymbol{\Gamma}_{j}$ as long as $\boldsymbol{\Gamma}_{j}^{\mathrm{T}} \boldsymbol{\Gamma}_{j}=\mathbf{I}_{p_{j}}$ and $\mathcal{M}\left(\boldsymbol{\Gamma}_{j}\right)=\mathcal{M}\left(\mathbf{A}_{j}\right)$.

To discover the latent segmentation, we need to estimate $\mathbf{A}=\left(\mathbf{A}_{1}, \ldots, \mathbf{A}_{q}\right)$, or more precisely, to estimate linear spaces $\mathcal{M}\left(\mathbf{A}_{1}\right), \ldots, \mathcal{M}\left(\mathbf{A}_{q}\right)$. To this end, we introduce some notation first. For any integer $k$, let $\boldsymbol{\Sigma}_{y}(k)=\operatorname{Cov}\left(\mathbf{y}_{t+k}, \mathbf{y}_{t}\right)$ and $\boldsymbol{\Sigma}_{x}(k)=\operatorname{Cov}\left(\mathbf{x}_{t+k}, \mathbf{x}_{t}\right)$. For a prescribed positive integer $k_{0}$, define

$$
\begin{aligned}
& \mathbf{W}_{y}=\sum_{k=0}^{k_{0}} \boldsymbol{\Sigma}_{y}(k) \boldsymbol{\Sigma}_{y}(k)^{\mathrm{T}}=\mathbf{I}_{p}+\sum_{k=1}^{k_{0}} \boldsymbol{\Sigma}_{y}(k) \boldsymbol{\Sigma}_{y}(k)^{\mathrm{T}}, \\
& \mathbf{W}_{x}=\sum_{k=0}^{k_{0}} \boldsymbol{\Sigma}_{x}(k) \boldsymbol{\Sigma}_{x}(k)^{\mathrm{T}}=\mathbf{I}_{p}+\sum_{k=1}^{k_{0}} \boldsymbol{\Sigma}_{x}(k) \boldsymbol{\Sigma}_{x}(k)^{\mathrm{T}} .
\end{aligned}
$$

Then both $\boldsymbol{\Sigma}_{x}(k)$ and $\mathbf{W}_{x}$ are block-diagonal, and

$$
\mathbf{W}_{y}=\mathbf{A} \mathbf{W}_{x} \mathbf{A}^{\mathrm{T}} \text {. }
$$

Note that both $\mathbf{W}_{y}$ and $\mathbf{W}_{x}$ are positive definite matrices. Let

$$
\mathbf{W}_{x} \boldsymbol{\Gamma}_{x}=\boldsymbol{\Gamma}_{x} \mathbf{D}
$$

that is, $\boldsymbol{\Gamma}_{x}$ is a $p \times p$ orthogonal matrix with the columns being the orthonormal eigenvectors of $\mathbf{W}_{x}$, and $\mathbf{D}$ is a diagonal matrix with the corresponding eigenvalues as the elements on the main diagonal. Then (2.6) implies that $\mathbf{W}_{y} \mathbf{A} \boldsymbol{\Gamma}_{x}=\mathbf{A} \boldsymbol{\Gamma}_{x} \mathbf{D}$. Hence the columns of $\boldsymbol{\Gamma}_{y} \equiv \mathbf{A} \boldsymbol{\Gamma}_{x}$ are the orthonormal eigenvectors of $\mathbf{W}_{y}$. Consequently,

$$
\boldsymbol{\Gamma}_{y}^{\mathrm{T}} \mathbf{y}_{t}=\boldsymbol{\Gamma}_{x}^{\mathrm{T}} \mathbf{A}^{\mathrm{T}} \mathbf{y}_{t}=\boldsymbol{\Gamma}_{x}^{\mathrm{T}} \mathbf{x}_{t},
$$

the last equality follows from (2.1). Put

$$
\mathbf{W}_{x}=\operatorname{diag}\left(\mathbf{W}_{x, 1}, \ldots, \mathbf{W}_{x, q}\right) .
$$

Then $\mathbf{W}_{x, j}$ is a $p_{j} \times p_{j}$ positive definite matrix, and the eigenvalues of $\mathbf{W}_{x, j}$ are also the eigenvalues of $\mathbf{W}_{x}$. Suppose that $\mathbf{W}_{x, i}$ and $\mathbf{W}_{x, j}$ do not share the same eigenvalues for any $i \neq j$. Then if we line up the eigenvalues of $\mathbf{W}_{x}$ (i.e., the eigenvalues of $\mathbf{W}_{x, 1}, \ldots, \mathbf{W}_{x, q}$ combining together) in the main diagonal of $\mathbf{D}$ according to the order of the blocks in $\mathbf{W}_{x}, \boldsymbol{\Gamma}_{x}$ must be a block-diagonal orthogonal matrix of the same shape as $\mathbf{W}_{x}$; see Proposition 1(i). However, the order of the eigenvalues is latent, and any $\boldsymbol{\Gamma}_{x}$ defined by (2.7) is nevertheless a columnpermutation (i.e., a matrix consisting of the same column vectors but arranged in a different order) of such a block-diagonal orthogonal matrix; see Proposition 1(ii). Hence each component of $\boldsymbol{\Gamma}_{x}^{\mathrm{T}} \mathbf{x}_{t}$ is a linear transformation of the elements in one of 
the $q$ subseries only, that is, the $p$ components of $\Gamma_{y}^{\mathrm{T}} \mathbf{y}_{t}=\Gamma_{x}^{\mathrm{T}} \mathbf{x}_{t}$ can be partitioned into the $q$ groups such that there exist neither contemporaneous nor serial correlations across different groups. Thus $\Gamma_{y}^{\mathrm{T}} \mathbf{y}_{t}$ can be regarded as a permutation of $\mathbf{x}_{t}$, and $\boldsymbol{\Gamma}_{y}$ can be viewed as a column-permutation of $\mathbf{A}$; see the discussion below (2.4). This leads to the following two-step estimation for $\mathbf{A}$ and $\mathbf{x}_{t}$ :

Step 1. Let $\widehat{\mathbf{S}}$ be an estimator for $\mathbf{W}_{y}$. Calculate a $p \times p$ orthogonal matrix $\widehat{\boldsymbol{\Gamma}}_{y}$ with the columns being the orthonormal eigenvectors of $\widehat{\mathbf{S}}$.

Step 2. The columns of $\widehat{\mathbf{A}}=\left(\widehat{\mathbf{A}}_{1}, \ldots, \widehat{\mathbf{A}}_{q}\right)$ are a permutation of the columns of $\widehat{\boldsymbol{\Gamma}}_{y}$ such that $\widehat{\mathbf{x}}_{t}=\widehat{\mathbf{A}}^{\mathrm{T}} \mathbf{y}_{t}$ is segmented into $q$ uncorrelated subseries $\widehat{\mathbf{x}}_{t}^{(j)}=\widehat{\mathbf{A}}_{j}^{\mathrm{T}} \mathbf{y}_{t}$, $j=1, \ldots, q$.

Step 1 is the key, as it provides an estimator for $\mathbf{A}$ except that the columns of the estimator are not grouped together according to the latent segmentation. The estimator $\widehat{\mathbf{S}}$ should be consistent, and will be constructed under various scenarios in Section 3 below. The permutation in Step 2 above can be carried out in principle by visual observation: plot cross correlogram of $\widehat{\mathbf{z}}_{t} \equiv \widehat{\boldsymbol{\Gamma}}_{y}^{\mathrm{T}} \mathbf{y}_{t}$ (using, e.g., $R$-function acf); see Figure 1(b). We then put those components of $\widehat{\mathbf{z}}_{t}$ together when there exist significant cross-correlations (at any lags) between those component series. Then $\widehat{\mathbf{A}}$ is obtained by rearranging the order of the columns of $\widehat{\boldsymbol{\Gamma}}_{y}$ accordingly.

REMARK 1. (i) Appropriate precaution should be exercised in the visual observation stated above. First, the visual observation become impractical when $p$ is large. Furthermore, most correlogram plots produced by statistical packages (including $R$ ) use the confidence bounds at $\pm 1.96 / \sqrt{n}$ for sample cross-correlations of two time series. Unfortunately, those bounds are only valid if at least one of the two series is white noise. In general, the confidence bounds depend on the autocorrelations of the two series. See Theorem 7.3.1 of Brockwell and Davis (1996). In Section 2.2, we will describe how the permutation can be performed without the benefit of visual observation for the cross correlogram of $\widehat{\mathbf{z}}_{t}$. Ledoit and Wolf (2004) and Paparoditis and Politis (2012) provide more modern approaches to view correlations.

(ii) $\mathbf{W}_{y}$ defined in (2.5) combines the information over different time lags together. In practice, we need to specify the integer $k_{0}$. Note that all terms on the right-hand side of (2.5) are nonnegative definite. Hence there is no information cancellation over different lags. This makes the method insensitive to the choice of $k_{0}$. In practice, a small $k_{0}$ is often sufficient, as long as the first $k_{0}$ lags carry sufficient information on the latent block diagonal structure even when the auto/crosscorrelations beyond lag $k_{0}$ are still significant. The examples in Section 4 lend further support to this assertion.

Proposition 1. (i) The orthogonal matrix $\boldsymbol{\Gamma}_{x}$ in (2.7) can be taken as a block-diagonal orthogonal matrix with the same block structure as $\mathbf{W}_{x}$. 
(ii) An orthogonal matrix $\boldsymbol{\Gamma}_{x}$ satisfies (2.7) if and only if its columns are a permutation of the columns of a block-diagonal orthogonal matrix described in (i), provided that any two different blocks $\mathbf{W}_{x, i}$ and $\mathbf{W}_{x, j}$ do not share the same eigenvalues.

Proposition 1(ii) requires that the $q$ blocks of $\mathbf{W}_{x}$ do not share the same eigenvalue(s). However, it does not rule out the possibility that each block $\mathbf{W}_{x, j}$ may have multiple eigenvalues. When different blocks share the same eigenvalue(s), Proposition 1 still holds with $\mathbf{W}_{x}$ replaced by $\mathbf{W}_{x}^{\star}$ which is also a block diagonal matrix with fewer than $q$ blocks obtained by combining together those $\mathbf{W}_{x, j}$ 's sharing at least one common eigenvalue into one larger block. This means that the proposed method will not be able to separate, for example, $\mathbf{x}_{t}^{(1)}$ and $\mathbf{x}_{t}^{(2)}$ if $\mathbf{W}_{x, 1}$ and $\mathbf{W}_{x, 2}$ share at least one common eigenvalue.

\subsection{Permutation.}

2.2.1. Permutation rule. The columns of $\widehat{\mathbf{A}}$ are a permutation of the columns of $\widehat{\boldsymbol{\Gamma}}_{y}$. The permutation is determined by grouping the components of $\widehat{\mathbf{z}}_{t}=\widehat{\boldsymbol{\Gamma}}_{y}^{\mathrm{T}} \mathbf{y}_{t}$ into $q$ groups, where $q$ and the cardinal numbers of those groups are unknown. Write $\widehat{\mathbf{z}}_{t}=\left(\widehat{z}_{1, t}, \ldots, \widehat{z}_{p, t}\right)^{\mathrm{T}}$. Let $\rho_{i, j}(h)$ denote the cross correlation between the two component series $\widehat{z}_{i, t}$ and $\widehat{z}_{j, t}$ at lag $h$. We say $\widehat{z}_{i, t}$ and $\widehat{z}_{j, t}$ connected if the multiple null hypothesis

$$
H_{0}: \rho_{i, j}(h)=0 \quad \text { for any } h=0, \pm 1, \pm 2, \ldots, \pm m
$$

is rejected, where $m \geq 1$ is a prescribed integer. Thus there exists significant evidence indicating nonzero correlations between two connected component series. Hence those components should be put in the same group. We may take $m=20$, or $m$ sufficiently large but smaller than $n / 4$, in the spirit of the rule of thumb proposed by Box and Jenkins [(1970), page 30], as we exclude long memory processes in this paper. Note that the autocorrelations of stationary (causal) VARMA processes decay exponentially fast. The permutation in Step 2 in Section 2.1 can be performed as follows:

i. Start with the $p$ groups with each group containing one component of $\widehat{\mathbf{z}}_{t}$ only.

ii. Combine two groups together if one connected pair are split over the two groups.

iii. Repeat Step ii above until all connected pairs are within one group.

We introduce below two methods for identifying the connected pair components of $\widehat{\mathbf{z}}_{t}=\widehat{\boldsymbol{\Gamma}}_{y}^{\mathrm{T}} \mathbf{y}_{t}$. 
2.2.2. Maximum cross correlation method. One natural way to test hypothesis $H_{0}$ in (2.10) is to use the maximum cross correlation over the lags between $-m$ and $m$ :

$$
\widehat{L}_{n}(i, j)=\max _{|h| \leq m}\left|\widehat{\rho}_{i, j}(h)\right|,
$$

where $\widehat{\rho}_{i, j}(h)$ is the sample cross correlation between $\widehat{z}_{i, t}$ and $\widehat{z}_{j, t}$ at lag $h$. We would reject $H_{0}$ for the pair $\left(\widehat{z}_{i, t}, \widehat{z}_{j, t}\right)$ if $\widehat{L}_{n}(i, j)$ is greater than an appropriate threshold value.

Instead of conducting multiple tests for each of the $p_{0} \equiv p(p-1) / 2$ pairs components of $\widehat{\mathbf{z}}_{t}$, we propose a ratio-based statistic to single out those pairs for which $H_{0}$ will be rejected. To this end, we rearrange the $p_{0}$ obtained $\widehat{L}_{n}(i, j)$ 's in the descending order: $\widehat{L}_{1} \geq \cdots \geq \widehat{L}_{p_{0}}$. Define

$$
\widehat{r}=\arg \max _{1 \leq j<c_{0} p_{0}} \widehat{L}_{j} / \widehat{L}_{j+1},
$$

where $c_{0} \in(0,1)$ is a prescribed constant. In all the numerical examples in Section 4 and the supplementary material [Chang, Guo and Yao (2018)], we use $c_{0}=0.75$. We reject $H_{0}$ for the pairs corresponding to $\widehat{L}_{1}, \ldots, \widehat{L}_{\widehat{r}}$.

The intuition behind this approach is as follows. Suppose among in total $p_{0}$ pairs of the components of $\mathbf{x}_{t}$ there are $r$ connected pairs only. Arrange the true maximum cross correlations in the descending order: $L_{1} \geq \cdots \geq L_{p_{0}}$. Then $L_{r}>0$ and $L_{r+1}=0$, and the ratio $L_{j} / L_{j+1}$ takes value $\infty$ for $j=r$. This motivates the estimator $\widehat{r}$ defined in (2.12) in which we exclude some minimum $\widehat{L}_{j}$ in the search for $\widehat{r}$ as $c_{0} \in(0,1)$. This is to avoid the fluctuations due to the ratios of extremely small values. This causes little loss in information as, for example, $0.75 p_{0}$ connected pairs would likely group most, if not all, component series together; see, for example, Example 2 in Section 4. The similar idea has been used in defining the factor dimensions in Lam and Yao (2012) and Chang, Guo and Yao (2015).

To state the asymptotic property of the above approach, we use a graph representation. Let the graph contain $p$ vertexes $\widehat{V}=\{1, \ldots, p\}$, representing $p$ component series of $\widehat{\mathbf{z}}_{t}$. Define an edge connecting vertexes $i$ and $j$ if $H_{0}$ in (2.10) for $\left(\widehat{z}_{i, t}, \widehat{z}_{j, t}\right)$ is rejected by the above ratio method. Let $\widehat{E}_{n}$ be the set consisting all those edges. Let $V=\{1, \ldots, p\}$ represent the $p$ component series of $\mathbf{z}_{t}=\boldsymbol{\Gamma}_{y}^{\mathrm{T}} \mathbf{y}_{t}$ defined in (2.8), and write $\mathbf{z}_{t}=\left(z_{1, t}, \ldots, z_{p, t}\right)^{\mathrm{T}}$. Define

$$
E=\left\{(i, j): \max _{|h| \leq m}\left|\operatorname{Corr}\left(z_{i, t+h}, z_{j, t}\right)\right|>0,1 \leq i<j \leq p\right\} .
$$

Each $(i, j) \in E$ can be reviewed as an edge. The graph $\left(\widehat{V}, \widehat{E}_{n}\right)$ is a consistent estimate for the graph $(V, E)$; see Proposition 2 below. To avoid the technical difficulties in dealing with " $0 / 0$ ", we modify (2.12) as follows:

$$
\widehat{r}=\arg \max _{1 \leq j<p_{0}}\left(\widehat{L}_{j}+\delta_{n}\right) /\left(\widehat{L}_{j+1}+\delta_{n}\right),
$$


where $\delta_{n}>0$ is a small constant. Assume

$$
\min _{(i, j) \in E} \max _{|h| \leq m}\left|\operatorname{Corr}\left(z_{i, t+h}, z_{j, t}\right)\right| \geq \epsilon_{n}
$$

for some $\epsilon_{n}>0$ and $n \epsilon_{n}^{2} \rightarrow \infty$. Write

$$
\varpi_{n}=\min _{1 \leq i<j \leq q} \min _{\lambda \in \sigma\left(\mathbf{W}_{x, i}\right), \mu \in \sigma\left(\mathbf{W}_{x, j}\right)}|\lambda-\mu|,
$$

where $\mathbf{W}_{x, i}$ is defined in (2.9), $\sigma\left(\mathbf{W}_{x, i}\right)$ denotes the set consisting of all the eigenvalues of $\mathbf{W}_{x, i}$. Here, $\epsilon_{n}$ denotes the weakest signal to be identified in $E$, and $\varpi_{n}$ is the minimum difference between the eigenvalues from the different diagonal blocks in $\mathbf{W}_{x}$. Arrange the true maximum cross correlations of $\mathbf{z}_{t}$ in the descending order $L_{1} \geq \cdots \geq L_{p_{0}}$ and define

$$
\chi_{n}=\max _{1 \leq j<r-1} L_{j} / L_{j+1},
$$

where $r=|E|$. Recall that $\widehat{\mathbf{S}}$ is the estimator for $\mathbf{W}_{y}$ used in Step 1 in Section 2.1. Let

$$
\widehat{\boldsymbol{\Sigma}}_{y}(h)=\frac{1}{n} \sum_{t=1}^{n-h}\left(\mathbf{y}_{t+h}-\overline{\mathbf{y}}\right)\left(\mathbf{y}_{t}-\overline{\mathbf{y}}\right)^{\mathrm{T}} \quad \text { and } \quad \overline{\mathbf{y}}=\frac{1}{n} \sum_{t=1}^{n} \mathbf{y}_{t} .
$$

Now we state the consistency in Proposition 2, which requires $\varpi_{n}>0$ [see Proposition 1(ii)]. The proof of Proposition 2 is similar to that of Theorem 2.4 of Chang, Guo and Yao (2015), and is therefore omitted.

Proposition 2. Let $\chi_{n} \delta_{n}=o\left(\epsilon_{n}\right)$ and $\varpi_{n}^{-1}\left\|\widehat{\mathbf{S}}-\mathbf{W}_{y}\right\|_{2}=o_{p}\left(\delta_{n}\right)$. Let the singular values of $\widehat{\boldsymbol{\Sigma}}_{y}(h)$ be uniformly bounded away from $\infty$ for all $|h| \leq m$. Then for $\widehat{r}$ defined in (2.13), it holds that $\mathbb{P}\left(\widehat{E}_{n}=E\right) \rightarrow 1$.

REMARK 2. (i) The inserting of $\delta_{n}$ in the definition of $\widehat{r}$ in (2.13) is to avoid the undetermined " $0 / 0$ " cases. In practice, we use $\widehat{r}$ defined by (2.12) instead, but with the search restricted to $1 \leq j \leq c_{0} p_{0}$, as $\delta_{n}$ subscribed in Proposition 2 is unknown. The simulation results reported in the supplementary material [Chang, Guo and Yao (2018)] indicate that (2.12) works reasonably well. See also Lam and Yao (2012) and Chang, Guo and Yao (2015).

(ii) The uniform boundedness for the singular values of $\widehat{\boldsymbol{\Sigma}}_{y}(h)$ was used to simplify the presentation. If $\max _{|h| \leq m}\left\|\widehat{\boldsymbol{\Sigma}}_{y}(h)\right\|_{2}=O_{p}\left(v_{n}\right)$ for some diverging $v_{n}$, we require the condition $\varpi_{n}^{-1} v_{n}\left\|\widehat{\mathbf{S}}-\mathbf{W}_{y}\right\|_{2}=o_{p}\left(\delta_{n}\right)$.

(iii) The finite sample performance can be improved by prewhitening each component series $\widehat{z}_{i, t}$ first. Then the asymptotic variance of $\widehat{\rho}_{i, j}(h)$ is $1 / n$ as long as $\operatorname{Corr}\left(z_{i, t+h}, z_{j, t}\right)=0$; see Corollary 7.3.1 of Brockwell and Davis (1996). This makes the maximum cross correlations for different pairs more comparable. Note that two weakly stationary time series are correlated if and only if their prewhitened series are correlated. 
2.2.3. FDR based on multiple tests. Alternatively, we can identify the connected pair components of $\widehat{\mathbf{z}}_{t}$ by a false discovery rate (FDR) procedure built on the multiple tests for cross correlations of each pair series.

In the same spirit of Remark 2(iii), we first prewhiten each component series of $\widehat{\mathbf{z}}_{t}$ separately, and then look into the cross correlations of the prewhitened series which are white noise. Thus we only need to test hypothesis (2.10) for two white noise series.

To fix the idea, let $\xi_{t}$ and $\eta_{t}$ denote two white noise series. Let $\rho(h)=$ $\operatorname{Corr}\left(\xi_{t+h}, \eta_{t}\right)$ and $\widehat{\rho}(h)$ be its sample analogue. By Theorem 7.3.1 of Brockwell and Davis (1996), $\widehat{\rho}\left(h_{1}\right)$ and $\widehat{\rho}\left(h_{2}\right)$, for any $h_{1} \neq h_{2}$, are asymptotically independent as $n \rightarrow \infty$, provided that $\rho(h)=0$ for all $h$, and the underlying processes are Gaussian. Hence the $P$-value for testing a simple null hypothesis $\rho(h)=0$ based on statistic $\widehat{\rho}(h)$ is approximately equal to $p_{h}=2 \Phi\{-\sqrt{n}|\widehat{\rho}(h)|\}$, where $\Phi(\cdot)$ denotes the distribution function of $N(0,1)$. Let $p_{(1)} \leq \cdots \leq p_{(2 m+1)}$ be the order statistics of $\left\{p_{h}: h=0, \pm 1, \ldots, \pm m\right\}$. As these $P$-values are approximately independent for large $n$, a multiple test at the significant level $\alpha \in(0,1)$ rejects $H_{0}$, defined in (2.10), if $p_{(j)} \leq j \alpha /(2 m+1)$ for at least one $1 \leq j \leq 2 m+1$; see Simes (1986) for details. Sarkar and Chang (1997) showed that it is still a valid test at the level $\alpha$ if $\widehat{\rho}(h)$, for different $h$, are positive-dependent. Hence the $P$-value for this multiple test for the null hypothesis $H_{0}$ is $P=\min _{1 \leq j \leq 2 m+1} p_{(j)}(2 m+1) / j$. The prewhitening is necessary in conducting the multiple test above, as otherwise $\widehat{\rho}\left(h_{1}\right)$ and $\widehat{\rho}\left(h_{2}\right)\left(h_{1} \neq h_{2}\right)$ are not asymptotically independent.

We can calculate the $P$-value for testing $H_{0}$ in (2.10) for each pair of the components of $\widehat{\mathbf{z}}_{t}$, resulting in the total $p_{0} \equiv p(p-1) / 2 P$-values. Arranging those $P$-values in ascending order: $P_{(1)} \leq \cdots \leq P_{\left(p_{0}\right)}$. Let

$$
d=\max \left\{k: 1 \leq k \leq p_{0}, P_{(k)} \leq k \beta / p_{0}\right\}
$$

for a given small $\beta \in(0,1)$. Then the FDR procedure with the error rate controlled under $\beta$ rejects the hypothesis $H_{0}$ for the $d$ pairs of the components of $\widehat{\mathbf{z}}_{t}$ corresponding to the $P$-values $P_{(1)}, \ldots, P_{(d)}$, that is, those $d$ pairs of components are connected. Since the $P$-values $P_{j}$ 's are no longer independent, the $\beta$ in (2.16) no longer admits the standard FDR interpretation. Nevertheless the $P$-values $P_{(1)}, \ldots, P_{(d)}$ give another way (in addition to the maximum cross correlation) to rank the pairs of the components of $\widehat{\mathbf{z}}_{t}$ according to the strength of the cross correlations. In fact, the ranking of the pairs in terms of the correlation strength matters most as far as the dimension-reduction is concerned; see, for example, Table 2 for Example 2 in Section 4. Different segmentations resulting from using different tuning parameters are caused effectively by how many those small (maybe still significant) correlations being used in determining a segmentation. The impact on, for example, post-sample forecasting is almost negligible; see Table 1 in Section 4. 
3. Theoretical properties. To gain more appreciation of the new methodology, we will show that there exists a permutation transformation which permutes the column vectors of $\widehat{\boldsymbol{\Gamma}}_{y}$, and the resulting new orthogonal matrix, denoted as $\widehat{\mathbf{A}}=\left(\widehat{\mathbf{A}}_{1}, \ldots, \widehat{\mathbf{A}}_{q}\right)$, is an adequate estimator for the transformation matrix $\mathbf{A}$ in (2.1) in the sense that $\mathcal{M}\left(\widehat{\mathbf{A}}_{j}\right)$ is consistent to $\mathcal{M}\left(\mathbf{A}_{j}\right)$ for each $j=1, \ldots, q$. Note that the columns of $\widehat{\boldsymbol{\Gamma}}_{y}$ are the $p$ orthonormal eigenvectors of the estimator $\widehat{\mathbf{S}}$ for $\mathbf{W}_{y}$; see Step 1 of the proposed method in Section 2.1. In this section, we treat this permutation transformation as an "oracle". In practice, it is identified either by a visual observation or by the methods presented in Section 2.2. Our goal here is to show that $\widehat{\boldsymbol{\Gamma}}_{y}$ is a valid estimator for $\mathbf{A}$ up to a column permutation. We establish the consistency under three different asymptotic modes: (i) the dimension $p$ is fixed, (ii) $p=o\left(n^{c}\right)$ and (iii) $\log p=o\left(n^{c}\right)$, as the sample size $n \rightarrow \infty$, where $c>0$ is a small constant. The convergence rates derived reflect the asymptotic orders of the estimation errors when $p$ is in different orders in relation to $n$.

To measure the errors in estimating $\mathcal{M}\left(\mathbf{A}_{j}\right)$, we adopt a metric on the Grassmann manifold of $r$-dimensional subspaces of $\mathbb{R}^{p}$ : for two $p \times r$ half orthogonal matrices $\mathbf{H}_{1}$ and $\mathbf{H}_{2}$ satisfying the condition $\mathbf{H}_{1}^{\mathrm{T}} \mathbf{H}_{1}=\mathbf{H}_{2}^{\mathrm{T}} \mathbf{H}_{2}=\mathbf{I}_{r}$, the distance between $\mathcal{M}\left(\mathbf{H}_{1}\right)$ and $\mathcal{M}\left(\mathbf{H}_{2}\right)$ is defined as

$$
D\left\{\mathcal{M}\left(\mathbf{H}_{1}\right), \mathcal{M}\left(\mathbf{H}_{2}\right)\right\}=\sqrt{1-r^{-1} \operatorname{tr}\left(\mathbf{H}_{1} \mathbf{H}_{1}^{\mathrm{T}} \mathbf{H}_{2} \mathbf{H}_{2}^{\mathrm{T}}\right)} .
$$

Then $\operatorname{D}\left\{\mathcal{M}\left(\mathbf{H}_{1}\right), \mathcal{M}\left(\mathbf{H}_{2}\right)\right\} \in[0,1]$. It is equal to 0 if and only if $\mathcal{M}\left(\mathbf{H}_{1}\right)=$ $\mathcal{M}\left(\mathbf{H}_{2}\right)$, and to 1 if and only if $\mathcal{M}\left(\mathbf{H}_{1}\right)$ and $\mathcal{M}\left(\mathbf{H}_{2}\right)$ are orthogonal; see, for example, Stewart and Sun (1990) and Pan and Yao (2008).

We always assume that the weakly stationary process $\mathbf{y}_{t}$ is $\alpha$-mixing, that is, its mixing coefficients $\alpha_{k, p} \rightarrow 0$ as $k \rightarrow \infty$, where

$$
\alpha_{k, p}=\sup _{i} \sup _{A \in \mathcal{F}_{-\infty}^{i}, B \in \mathcal{F}_{i+k}^{\infty}}|\mathbb{P}(A \cap B)-\mathbb{P}(A) \mathbb{P}(B)|,
$$

and $\mathcal{F}_{i}^{j}$ is the $\sigma$-field generated by $\left\{\mathbf{y}_{t}: i \leq t \leq j\right\}$. In sequel, we denote by $\sigma_{i, j}^{(k)}$ the $(i, j)$ th element of $\Sigma_{y}(k)$ for each $i, j=1, \ldots, p$ and $k=1, \ldots, k_{0}$. The $\alpha$-mixing is a mild condition on "asymptotic independence". Many time series including causal ARMA processes with continuously distributed innovations are $\alpha$-mixing with exponentially decaying mixing coefficients; see, for example, Section 2.6.1 of Fan and Yao (2003) and the references within. Nevertheless, it rules out, for example, long memory processes.

We use the notation $\boldsymbol{\mu}=\mathbb{E}\left(\mathbf{y}_{t}\right), \mathbf{y}_{t}=\left(y_{1, t}, \ldots, y_{p, t}\right)^{\mathrm{T}}$ and $\boldsymbol{\mu}=\left(\mu_{1}, \ldots, \mu_{p}\right)^{\mathrm{T}}$.

3.1. Asymptotics when $n \rightarrow \infty$ and p fixed. When the dimension $p$ is fixed, we estimate $\mathbf{W}_{y}$ defined in (2.5) by the plug-in estimator

$$
\widehat{\mathbf{S}}=\mathbf{I}_{p}+\sum_{k=1}^{k_{0}} \widehat{\boldsymbol{\Sigma}}_{y}(k) \widehat{\boldsymbol{\Sigma}}_{y}(k)^{\mathrm{T}},
$$


where $\widehat{\boldsymbol{\Sigma}}_{y}(k)$ is defined in (2.15). We show that the standard $\sqrt{n}$ convergence rate prevails as now $p$ is fixed. We introduce some regularity conditions first.

CONDITION 1. It holds that $\sup _{t} \max _{1 \leq i \leq p} \mathbb{E}\left(\left|y_{i, t}-\mu_{i}\right|^{2 \gamma}\right) \leq K_{1}$ for some constants $\gamma>2$ and $K_{1}>0$.

CONDITION 2. The mixing coefficients $\alpha_{k, p}$ defined in (3.1) satisfy the condition $\sum_{k=1}^{\infty} \alpha_{k, p}^{1-2 / \gamma}<\infty$, where $\gamma>2$ is given in Condition 1 .

THEOREM 1. Let Conditions 1 and 2 hold, $p$ be fixed, and $\varpi_{n}$ in (2.14) be positive. Then $\max _{1 \leq j \leq q} D\left\{\mathcal{M}\left(\widehat{\mathbf{A}}_{j}\right), \mathcal{M}\left(\mathbf{A}_{j}\right)\right\}=O_{p}\left(n^{-1 / 2}\right)$, where the columns of $\widehat{\mathbf{A}}=\left(\widehat{\mathbf{A}}_{1}, \ldots, \widehat{\mathbf{A}}_{q}\right)$ are a permutation of the $p$ orthonormal eigenvectors of $\widehat{\mathbf{S}}$ defined in (3.2).

REMARK 3. This result can be extended to a nonstationary case. For $p$ dimensional nonstationary time series $\mathbf{y}_{t}$, we assume that $\mathbf{y}_{t}=\mathbf{A} \mathbf{x}_{t}$ where $\mathbf{x}_{t}$ satisfies (2.2). Let $\boldsymbol{\Sigma}_{y}(k)=(n-k)^{-1} \sum_{t=1}^{n-k} \operatorname{Cov}\left(\mathbf{y}_{t+k}, \mathbf{y}_{t}\right)$ and $\boldsymbol{\Sigma}_{x}(k)=(n-$ $k)^{-1} \sum_{t=1}^{n-k} \operatorname{Cov}\left(\mathbf{x}_{t+k}, \mathbf{x}_{t}\right)$, which can be viewed as the extension of the conventional autocovariance for stationary process to nonstationary case. Then (2.6) still holds. Following the same arguments as in Chang, Guo and Yao (2015), it can be shown that there exists $\widehat{\mathbf{A}}=\left(\widehat{\mathbf{A}}_{1}, \ldots, \widehat{\mathbf{A}}_{q}\right)$ such that Theorem 1 holds, where the columns of $\widehat{\mathbf{A}}$ are a permutation of the $p$ orthonormal eigenvectors of $\widehat{\mathbf{S}}$ defined in (3.2) with $\widehat{\boldsymbol{\Sigma}}_{y}(k)$ specified in (2.15).

3.2. Asymptotics when $n \rightarrow \infty$ and $p=o\left(n^{c}\right)$. In the contemporary statistics dealing with large data, conventional wisdom assumes that $p$ diverges together with $n$. Since $\left\|\widehat{\mathbf{S}}-\mathbf{W}_{y}\right\|_{F}=O_{p}\left(p n^{-1 / 2}\right)$ for $\widehat{\mathbf{S}}$ defined in (3.2), it is necessary that $p=o\left(n^{1 / 2}\right)$ in order to retain the consistency (but with a slower convergence rate than $\sqrt{n})$. This means that $p$ can only be as large as $p=o\left(n^{1 / 2}\right)$ if we do not entertain any additional assumptions on the underlying structure. In order to deal with large $p$, we impose a sparsity condition on the transformation matrix $\mathbf{A}$ first.

Condition 3. For $\mathbf{A}=\left(a_{i, j}\right)$ in (2.1), $\max _{1 \leq j \leq p} \sum_{i=1}^{p}\left|a_{i, j}\right|^{\iota} \leq s_{1}$ and $\max _{1 \leq i \leq p} \sum_{j=1}^{p}\left|a_{i, j}\right|^{\iota} \leq s_{2}$, for some constant $\iota \in[0,1)$, where $s_{1}$ and $s_{2}$ may diverge together with $p$.

When $p$ is fixed, Condition 3 holds for $s_{1}=s_{2}=p$ and any $\iota \in[0,1)$, as $\mathbf{A}$ is an orthogonal matrix. For large $p, s_{1}$ and $s_{2}$ control the degree of the sparsity of the columns and the rows of $\mathbf{A}$, respectively. A small $s_{1}$ entails that each component series of $\mathbf{x}_{t}$ only contributes to a small fraction of the components of $\mathbf{y}_{t}$. A small $s_{2}$ entails that each component of $\mathbf{y}_{t}$ is a linear combination of a small number of the components of $\mathbf{x}_{t}$. The sparsity of $\mathbf{A}$ is also controlled by constant $\iota$ : the smaller $\iota$ 
is, the more sparse $\mathbf{A}$ is. We will show that the stronger sparsity leads to the faster convergence for our estimator; see Remark 4(ii) below.

If $p$ diverges faster than $n^{1 / 2}$, the sample autocovariance matrix $\widehat{\boldsymbol{\Sigma}}_{y}(k)=$ $\left(\widehat{\sigma}_{i, j}^{(k)}\right)_{p \times p}$, given in (2.15), is no longer a consistent estimator for $\boldsymbol{\Sigma}_{y}(k)$. Inheriting the spirit of threshold estimator for large covariance matrix by Bickel and Levina (2008), we employ the following threshold estimator instead:

$$
T_{u}\left\{\widehat{\boldsymbol{\Sigma}}_{y}(k)\right\}=\left(\widehat{\sigma}_{i, j}^{(k)} \mathbb{I}\left\{\left|\widehat{\sigma}_{i, j}^{(k)}\right| \geq u\right\}\right)_{p \times p},
$$

where $\mathbb{I}(\cdot)$ is the indicator function, $u>0$ sets the threshold level. Lemma 4 of Chang, Guo and Yao (2018) implies that $\max _{1 \leq i, j \leq p}\left|\widehat{\sigma}_{i, j}^{(k)}-\sigma_{i, j}^{(k)}\right|=$ $O_{p}\left(\max \left\{p^{2 / l} n^{-(l-1) / l},\left(n^{-1} \log p\right)^{1 / 2}\right\}\right)$ for $l$ specified in Conditions 4 and 5 later. Hence we set the threshold at $u=\vartheta_{n}$, where

$$
\vartheta_{n}=M \max \left\{p^{2 / l} n^{-(l-1) / l},\left(n^{-1} \log p\right)^{1 / 2}\right\},
$$

and $M>0$ is a constant. Consequently, we define now

$$
\widehat{\mathbf{S}} \equiv \widehat{\mathbf{W}}_{y}^{(\text {thre })}=\mathbf{I}_{p}+\sum_{k=1}^{k_{0}} T_{u}\left\{\widehat{\boldsymbol{\Sigma}}_{y}(k)\right\} T_{u}\left\{\widehat{\boldsymbol{\Sigma}}_{y}(k)\right\}^{\mathrm{T}} .
$$

Lemma 7 in Chang, Guo and Yao (2018) shows that $\widehat{\mathbf{W}}_{y}^{\text {(thre) }}$ is a consistent estimator for $\mathbf{W}_{y}$, which requires a stronger version of Conditions 1 and 2 as now $p$ diverges together with $n$.

CONDITION 4. As $x \rightarrow \infty$, it holds that $\sup _{t} \max _{1 \leq i \leq p} \mathbb{P}\left(\left|y_{i, t}-\mu_{i}\right|>x\right)=$ $O\left\{x^{-2(l+\tau)}\right\}$ for some constants $l>2$ and $\tau>0$.

CONDITION 5. The mixing coefficients $\alpha_{k, p}$ given in (3.1) satisfy the condition $\sup _{p \geq 1} \alpha_{k, p}=O\left\{k^{-(l-1)(l+\tau) / \tau}\right\}$ as $k \rightarrow \infty$, where $l$ and $\tau$ are given in Condition 4.

Conditions 4 and 5 ensure the Fuk-Nagaev-type inequalities for $\alpha$-mixing processes; see Rio (2000) and Liu, Xiao and Wu (2013). Put

$$
\begin{aligned}
\rho_{j} & =\min _{i \neq j} \min _{\lambda \in \sigma\left(\mathbf{W}_{x, i}\right), \mu \in \sigma\left(\mathbf{W}_{x, j}\right)}|\lambda-\mu|, \quad j=1, \ldots, q, \\
\delta & =s_{1} s_{2} \max _{1 \leq j \leq q} p_{j} \quad \text { and } \quad \kappa=\max _{1 \leq k \leq k_{0}}\left\|\boldsymbol{\Sigma}_{x}(k)\right\|_{2} .
\end{aligned}
$$

TheOREM 2. Let Conditions 3, 4 and 5 hold, $p=o\left\{n^{(l-1) / 2}\right\}$, and $\min _{1 \leq j \leq q} \rho_{j}>0$ for $\rho_{j}$ defined in (3.6). Then

$$
\max _{1 \leq j \leq q} \rho_{j} D\left\{\mathcal{M}\left(\widehat{\mathbf{A}}_{j}\right), \mathcal{M}\left(\mathbf{A}_{j}\right)\right\}=O_{p}\left\{\kappa \vartheta_{n}^{1-\imath} \delta+\vartheta_{n}^{2(1-\imath)} \delta^{2}\right\}
$$


where the columns of $\widehat{\mathbf{A}}=\left(\widehat{\mathbf{A}}_{1}, \ldots, \widehat{\mathbf{A}}_{q}\right)$ are a permutation of the $p$ orthonormal eigenvectors of matrix $\widehat{\mathbf{S}}$ defined in (3.5) with the threshold $u=\vartheta_{n}$ given in (3.4) in which constant $l$ satisfies Conditions 4 and 5 .

REMARK 4. (i) Theorem 2 presents the uniform convergence rate for $\rho_{j} D\left\{\mathcal{M}\left(\widehat{\mathbf{A}}_{j}\right), \mathcal{M}\left(\mathbf{A}_{j}\right)\right\}$. As $\rho_{j}$ measures the minimum difference between the eigenvalues of $\mathbf{W}_{x, j}$ and those of the other blocks, it is intuitively clear that the smaller this difference is, more difficult the estimation for $\mathcal{M}\left(\mathbf{A}_{j}\right)$ is.

(ii) As $\boldsymbol{\Sigma}_{y}(k)=\mathbf{A} \boldsymbol{\Sigma}_{x}(k) \mathbf{A}^{\mathrm{T}}$, the largest block size $S_{\max }=\max _{1 \leq j \leq q} p_{j}$ and the sparsity of $\mathbf{A}$ determines the sparsity of $\boldsymbol{\Sigma}_{y}(k)$. Lemma 5 of Chang, Guo and Yao (2018) shows that the sparsity of $\boldsymbol{\Sigma}_{y}(k)$ can be evaluated by $\delta$ defined in (3.7). A small value of $S_{\max }$ represents a high degree of sparsity for $\boldsymbol{\Sigma}_{x}(k)$, and thus, also for $\boldsymbol{\Sigma}_{y}(k)$, while the sparsity of $\mathbf{A}$ is reflected by $\iota, s_{1}$ and $s_{2}$; see Condition 3 and the comments immediately below it. The convergence rates specified in Theorem 2 contain factors $\delta$ or $\delta^{2}$. Hence the more sparse $\boldsymbol{\Sigma}_{y}(k)$ is (i.e., the smaller $\delta$ is), the faster the convergence is.

(iii) With the sparsity imposed in Condition 3, the dimension of time series can be as large as $p=o\left\{n^{(l-1) / 2}\right\}$, where $l>2$ is determined by the tail probabilities described in Condition 4.

(iv) Similar to Theorem 1, the result in Theorem 2 can also be extended to nonstationary case; see Remark 3.

(v) Instead of Condition 3, we may impose the sparsity condition on each $\boldsymbol{\Sigma}_{y}(k)$ such as $\max _{1 \leq j \leq p} \sum_{i=1}^{p}\left|\sigma_{i, j}^{(k)}\right|^{l} \leq s_{3}$ and $\max _{1 \leq i \leq p} \sum_{j=1}^{p}\left|\sigma_{i, j}^{(k)}\right|^{\imath} \leq s_{3}$ for some $\iota \in[0,1)$. Then the convergence rate in Theorem 2 changes to $O_{p}\left\{\kappa \vartheta_{n}^{1-\iota} s_{3}+\right.$ $\left.\vartheta_{n}^{2(1-\iota)} s_{3}^{2}\right\}$. Under the ideal case $\kappa=O(1), \min _{1 \leq j \leq q} \rho_{j} \asymp q^{-1}$ and $s_{3} \asymp p^{\zeta}$ for some $\zeta \in[0,1)$, we have $\max _{1 \leq j \leq q} D\left\{\mathcal{M}\left(\widehat{\mathbf{A}}_{j}\right), \mathcal{M}\left(\mathbf{A}_{j}\right)\right\}=O_{p}\left(p^{\zeta} q \vartheta_{n}^{1-\iota}\right)$ provided that $p^{\zeta} \vartheta_{n}^{1-\iota}=O(1)$. Therefore, if $p^{\zeta} q \vartheta_{n}^{1-\iota}=o(1)$, we can estimate each subspace $\mathcal{M}\left(\mathbf{A}_{j}\right)$ consistently.

3.3. Asymptotics when $n \rightarrow \infty$ and $\log p=o\left(n^{c}\right)$. To handle the ultra highdimensional cases where $p$ grows at an exponential rate of $n$, we need following stronger conditions (than Conditions 4 and 5) on the decays of the tail probabilities of $\mathbf{y}_{t}$ and the mixing coefficients $\alpha_{k, p}$ defined in (3.1).

Condition 6. For any $x>0$ and $\|\mathbf{v}\|_{2}=1, \sup _{t} \mathbb{P}\left\{\left|\mathbf{v}^{\mathrm{T}}\left(\mathbf{y}_{t}-\boldsymbol{\mu}\right)\right|>x\right\} \leq$ $K_{2} \exp \left(-K_{3} x^{r_{1}}\right)$, where $K_{2}, K_{3}>0$, and $r_{1} \in(0,2]$ are constants.

Condition 7. For all $k \geq 1, \sup _{p \geq 1} \alpha_{k, p} \leq \exp \left(-K_{4} k^{r_{2}}\right)$, where $K_{4}>0$ and $r_{2} \in(0,1]$ are some constants.

Condition 6 requires the tail probabilities of linear combinations of $\mathbf{y}_{t}$ decay exponentially fast. When $r_{1}=2, \mathbf{y}_{t}$ is sub-Gaussian. It is also intuitively clear 
that the large $r_{1}$ and/or $r_{2}$ would only make Conditions 6 and/or 7 stronger. The restrictions $r_{1} \leq 2$ and $r_{2} \leq 1$ are introduced only for the presentation convenience, as Theorem 3 below applies to the ultra high-dimensional cases with

$$
\log p=o\left\{n^{\varrho /(2-\varrho)}\right\} \quad \text { where } \varrho=1 /\left(2 r_{1}^{-1}+r_{2}^{-1}\right) \text {. }
$$

We still use $\widehat{\mathbf{S}}=\widehat{\mathbf{W}}_{y}^{\text {(thre) }}$ defined in (3.5) in Step 1 of our procedure. But now the threshold value is set at $u=M\left(n^{-1} \log p\right)^{1 / 2}$ in (3.3), as Lemma 8 in Chang, Guo and Yao (2018) indicates that $\max _{1 \leq i, j \leq p}\left|\widehat{\sigma}_{i, j}^{(k)}-\sigma_{i, j}^{(k)}\right|=O_{p}\left\{\left(n^{-1} \log p\right)^{1 / 2}\right\}$ when $p$ is specified by (3.8). Recall that $\delta$ and $\kappa$ are defined in (3.7).

Theorem 3. Let Conditions 3, 6 and 7 hold, $\min _{1 \leq j \leq q} \rho_{j}>0$ for $\rho_{j}$ defined in (3.6), and p satisfy (3.8). Then

$$
\max _{1 \leq j \leq q} \rho_{j} D\left\{\mathcal{M}\left(\widehat{\mathbf{A}}_{j}\right), \mathcal{M}\left(\mathbf{A}_{j}\right)\right\}=O_{p}\left\{\kappa\left(n^{-1} \log p\right)^{(1-\imath) / 2} \delta+\left(n^{-1} \log p\right)^{1-\imath} \delta^{2}\right\}
$$

where the columns of $\widehat{\mathbf{A}}=\left(\widehat{\mathbf{A}}_{1}, \ldots, \widehat{\mathbf{A}}_{q}\right)$ are a permutation of the $p$ orthonormal eigenvectors of $\widehat{\mathbf{S}}$ defined in (3.5) with the threshold level $u \asymp\left(n^{-1} \log p\right)^{1 / 2}$.

Similar to Remark 4(v), we may also impose the sparsity condition on each $\boldsymbol{\Sigma}_{y}(k)$ such as $\max _{1 \leq j \leq p} \sum_{i=1}^{p}\left|\sigma_{i, j}^{(k)}\right|^{\iota} \leq s_{3}$ and $\max _{1 \leq i \leq p} \sum_{j=1}^{p}\left|\sigma_{i, j}^{(k)}\right|^{\iota} \leq$ $s_{3}$ for some $\iota \in[0,1)$. Then the convergence rate in Theorem 3 changes to $O_{p}\left\{\kappa\left(n^{-1} \log p\right)^{(1-\iota) / 2} s_{3}+\left(n^{-1} \log p\right)^{1-\iota} s_{3}^{2}\right\}$. Under the ideal case $\kappa=O(1)$ and $\min _{1 \leq j \leq q} \rho_{j} \asymp q^{-1}$, we have $\max _{1 \leq j \leq q} D\left\{\mathcal{M}\left(\widehat{\mathbf{A}}_{j}\right), \mathcal{M}\left(\mathbf{A}_{j}\right)\right\}=$ $O_{p}\left\{q\left(n^{-1} \log p\right)^{(1-\iota) / 2} s_{3}\right\}$ provided that $\left(n^{-1} \log p\right)^{1-\iota} s_{3}^{2}=O(1)$. Therefore, if $q^{2}\left(n^{-1} \log p\right)^{1-\iota} s_{3}^{2}=o(1)$, we can estimate $\mathcal{M}\left(\mathbf{A}_{j}\right)$ 's consistently.

4. Numerical properties. Two questions arise with the proposed methodology in this paper: (i) Is the segmentation assumption (2.1) and (2.2) of practical relevance? (ii) What would the proposed method lead to if the assumption does not hold? To answer these questions, we report below the illustration with four real data sets from different fields. Chang, Guo and Yao (2018) contains the illustration with simulated data.

We always standardize the data first, that is, to replace $\mathbf{y}_{t}$ by $\left\{\widehat{\boldsymbol{\Sigma}}_{y}(0)\right\}^{-1 / 2} \mathbf{y}_{t}$, where $\widehat{\Sigma}_{y}(0)$ is the sample covariance matrix (2.15) for Examples $1-3$, and is the truncated one for Example 4 [see (3.3)]. Then the segmentation transformation is $\widehat{\mathbf{x}}_{t}=\widehat{\mathbf{B}} \mathbf{y}_{t}$, where $\widehat{\mathbf{B}}=\widehat{\boldsymbol{\Gamma}}_{y}^{\mathrm{T}}\left\{\widehat{\boldsymbol{\Sigma}}_{y}(0)\right\}^{-1 / 2}$, and $\widehat{\boldsymbol{\Gamma}}_{y}$ is the $p \times p$ orthogonal matrix specified in Step 1 in Section 2.1 based on the new time series $\left\{\widehat{\boldsymbol{\Sigma}}_{y}(0)\right\}^{-1 / 2} \mathbf{y}_{t}$. We always prewhiten each transformed component series of $\widehat{\mathbf{x}}_{t}$ before applying the permutation methods described in Section 2.2. The prewhitening is carried out by fitting each series an AR model with the order between 0 and 5 determined by AIC. The resulting residual series is taken as a prewhitened series. We set the 
upper bound for the AR-order at 5 to avoid over-whitening with finite samples. We always set $c_{0}=0.75$ in (2.12) and $k_{0}=5$ in computing $\stackrel{\mathbf{S}}{\mathbf{S}}$ unless stated explicitly. See Remark 1(ii).

To show the advantages of the proposed TS-PCA transformation, we also conduct post-sample forecasting and compare the forecasts based on the original data directly and those via TS-PCA transformation. To ensure that the comparison is fair and objective, we adopt VAR models with the order determined by AIC for both the original and the transformed data, involving no fine-tuning on the form of model and the order determination. Note that there is no universally accepted optimal model for a real data set. We use the $R$-function VAR in the $R$-package Vars to fit VAR models. We also report the results from the restricted VAR model (RVAR) obtained by setting insignificant coefficients to 0 in a fitted VAR model, using the $R$-function restrict in the $R$-package vars.

Some useful tips from the real data analysis below are worth mentioning. First, the segmentation assumption is reasonable for Examples 1, 3 and 4. Second, when the segmentation assumption is invalid (Example 2), the TS-PCA transformation leads to approximate segmentations which also improve the forecasting performance. Third, when $p$ is large or moderately large, it is necessary to apply appropriate dimension-reduction techniques (such as TS-PCA) in order to take advantage from the dependence across different series (Examples 3 and 4). Finally, the forecasting via the TS-PCA transformation always outperform that directly based on the original data in all the real data examples. The reason for this is explained at the end of Section 6.

EXAMPLE 1 (Continued). We continue the analysis with the monthly temperature data in the 7 cities in China. The result reported in Section 2.1 was obtained with $k_{0}=5$ in (2.5). The profile of the segmentation is unchanged for $1 \leq k_{0} \leq 36$. For $p=7$, we do not need to apply the methods in Section 2.2 for permuting the transformed series. Nevertheless, exactly the same grouping is obtained by the permutation based on the maximum cross correlation method with $1 \leq m \leq 30$ in (2.10), or by the permutation based on FDR with $1 \leq m \leq 30$ and $0.001 \% \leq \beta \leq 1 \%$ in (2.16).

Forecasting the original time series $\mathbf{y}_{t}$ can be carried out in two steps. First, we forecast the components of $\widehat{\mathbf{x}}_{t}$ using 5 models according to the segmentation, that is, one VAR for the first three components, and a univariate AR model for each of the last four components. Then the forecasted values for $\mathbf{y}_{t}$ are obtained via the transformation $\widehat{\mathbf{y}}_{t}=\widehat{\mathbf{B}}^{-1} \widehat{\mathbf{x}}_{t}$. For each of the last 24 observations in this data set (i.e., the monthly temperatures in 1997 and 1998), we use the data up to the previous month to fit three forecasting models: the model based on the segmentation (which is a collection of 5 VAR/AR models for the 5 segmented subseries of $\widehat{\mathbf{x}}_{t}$ ), the VAR and RVAR models for the original data. We difference the original data at lag 12 before fitting them directly with VAR and RVAR models, to remove the seasonal components. For fitting the segmented series $\widehat{\mathbf{x}}_{t}$, we only difference its first two 
component series also at lag 12 since only they have seasonal components. The one-step-ahead forecasts can be obtained directly from the fitted models. The twostep-ahead forecasts are obtained based on the plug-in method, that is, using the one-step-ahead forecasted values as true values.

For each component series of $\mathbf{y}_{t}$, we calculate the mean squared predictive errors (MSPE) $d^{-1} \sum_{h=1}^{d}\left(\widehat{y}_{i, n_{0}+h}-y_{i, n_{0}+h}\right)^{2}$ for both one-step-ahead and two-stepahead forecasting, where $\widehat{y}_{i, n_{0}+h}$ denotes the associated forecast for $y_{i, n_{0}+h}$ (for this example, $d=24$ and $n_{0}=n-24$ ). The mean and standard deviations of those MSPEs over the 7 cities are listed in Table 1. Both the mean and standard deviation of the MSPEs based on TS-PCA are much smaller than those based on the direct VAR or RVAR models for original data. To evaluate the sensitivity of the segmentation, we also consider an over-segmentation case for $\widehat{\mathbf{x}}_{t}$ with 6 groups $(\{1,2\}$, $\{3\},\{4\},\{5\},\{6\},\{7\})$, and an incomplete-segmentation case with 4 groups $(\{1,2,3\},\{5,6\},\{4\},\{7\})$. Table 1 shows that, though the predictions for over-

TABLE 1

One-step and two-step ahead post-sample forecasting: means and standard deviations (in subscripted bracket) of MSPEs for Examples 1, 3 and 4 and means and standard deviations (in subscripted bracket) of the relative MSPEs for Example 2

\begin{tabular}{clcc}
\hline & \multicolumn{1}{c}{ Method } & One-step forecast & Two-step forecast \\
\hline Example 1 & VAR & $2.470_{(0.416)}$ & $2.559_{(0.385)}$ \\
$(p=7)$ & RVAR & $2.530_{(0.398)}$ & $2.615_{(0.382)}$ \\
& Segmentation with 5 groups & $2.221_{(0.339)}$ & $2.203_{(0.323)}$ \\
& Segmentation with 6 groups & $2.417_{(0.348)}$ & $2.419_{(0.326)}$ \\
& Segmentation with 4 groups & $2.421_{(0.343)}$ & $2.422_{(0.325)}$ \\
Example 2 & VAR & $0.950_{(0.148)}$ & $0.726_{(0.328)}$ \\
$(p=7)$ & RVAR & $0.962_{(0.138)}$ & $0.796_{(0.277)}$ \\
& Segmentation with 4 groups & $0.884_{(0.180)}$ & $0.708_{(0.377)}$ \\
& Segmentation with 7 groups & $0.919_{(0.130)}$ & $0.884_{(0.219)}$ \\
Example 3 & Segmentation with 3 groups & $0.873_{(0.176)}$ & $0.694_{(0.377)}$ \\
$(p=25)$ & Univariate AR & $0.208_{(0.551)}$ & $0.194_{(0.539)}$ \\
& VAR & $0.295_{(0.806)}$ & $0.301_{(0.855)}$ \\
& RVAR & $0.293_{(0.820)}$ & $0.296_{(0.863)}$ \\
& Segmentation with 24 groups & $0.153_{(0.134)}$ & $0.163_{(0.124)}$ \\
& Segmentation with 25 groups & $0.110_{(0.084)}$ & $0.132_{(0.091)}$ \\
Example 4 & Segmentation with 23 groups & $0.151_{(0.133)}$ & $0.159_{(0.121)}$ \\
$(p=84)$ & Univariate AR & $0.55_{(0.204)}$ & $0.835_{(0.284)}$ \\
& Segmentation with 83 groups & $0.485_{(0.185)}$ & $0.662_{(0.224)}$ \\
& Segmentation with 84 groups & $0.484_{(0.184)}$ & $0.662_{(0.224)}$ \\
& Segmentation with 50 groups & $0.492_{(0.187)}$ & $0.678_{(0.228)}$ \\
& Segmentation with 70 groups & $0.474_{(0.180)}$ & $0.664_{(0.225)}$ \\
\hline
\end{tabular}


and incomplete-segmentation are worse than the segmentation with the 5 groups, they still outperform both VAR and RVAR models.

EXAMPLE 2. We consider the weekly notified measles cases in 7 cities in England (i.e., London, Bristol, Liverpool, Manchester, Newcastle, Birmingham and Sheffield) in 1948-1965, before the advent of vaccination. All the 7 series show biennial cycles, which is a common feature in measles dynamics in the prevaccination period. This biennial cycling is the major driving force for the cross correlations among different component series displayed in Figure 2(a). The cross correlogram of the transformed data is displayed in Figure 2(b). Since none of the transformed component series are white noise, the confidence bounds in Figure 2(b) could be misleading; see Remark 1(i).

We apply prewhitening to each transformed component time series by fitting an AR model with the order determined by AIC. Although all those 7 filtered time series behave like white noise, there are still quite a few small but significant cross correlations here and there. Figure 3(a) plots, in descending order, the maximum cross correlations $\widehat{L}_{n}(i, j)$ defined in (2.11) for those 7 transformed and prewhitened series. As $1.96 / \sqrt{n}=0.064$ with $n=937$ now, one may argue that the segmentation assumption does not hold for this example. Consequently, the ratio estimator $\widehat{r}$ defined in (2.12) does not make any sense for this example; see also Figure 3(b).

Nevertheless Figure 3(a) ranks the pairs of transformed component series according to the strength of the cross correlation. If we would only accept $r$ connected pairs, this leads to an approximate segmentation according to the rule set in Section 2.2.1. By doing this, we effectively ignore some small, though still statistically significant, cross correlations. Table 2 lists the different segmentations corresponding to the different values of $r$. It shows that the group $\{4,5\}$ is always present until all the 7 series merge together. Further it only takes 6 connected pairs, corresponding to the 6 largest points in Figure 3(a), to merge all the series together.

The forecasting comparison is conducted in the same manner as in Example 1. We adopt the segmentation with 4 groups: $\{1,2,3\},\{4,5\},\{6\}$ and $\{7\}$, that is, we regard that only the three pairs, corresponding to the 3 maximum cross correlations in Figure 3(a), are connected. We forecast the notified measles cases in the last 14 weeks of the period for all the 7 cities. Due to the fact that the data from different cities are on different scales, we present the results based on relative MSPEs in Table 1: a relative MSPE is the ratio of a MSPE concerned over that obtained from fitting each original component series with an AR model. Once again the forecasting based on this (approximate) segmentation is much more accurate than those based on the direct VAR and RVAR models, although we have ignored quite a few small but significant cross correlations among the transformed series. Table 1 also reports an over-segmentation case with each transformed series as an individual group, and an alternative segmentation case with 3 groups $(\{1,2,3,7\}$, $\{4,5\},\{6\})$. The over-segmentation ignores all the correlations among different 
Y1

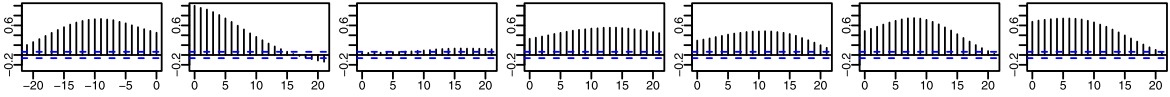

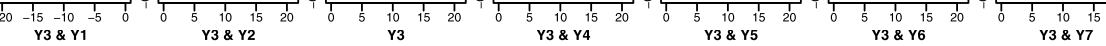

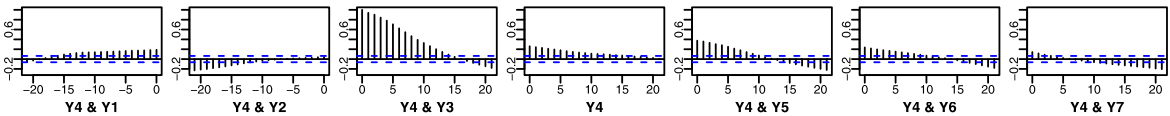
Y4 \& Y6
Y \& Y7

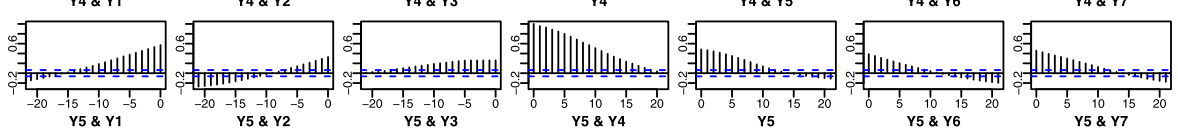
Y5 \& Y1

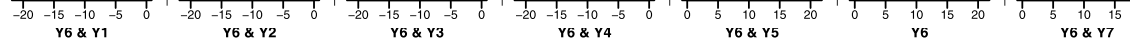

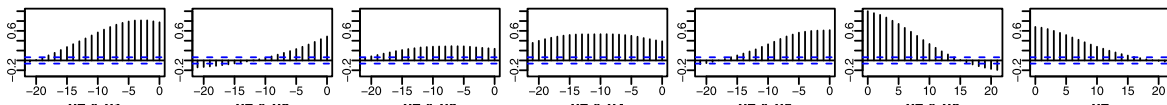

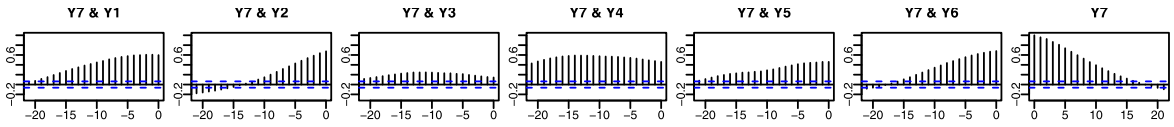
(a) Cross correlogram of the 7 original measles series
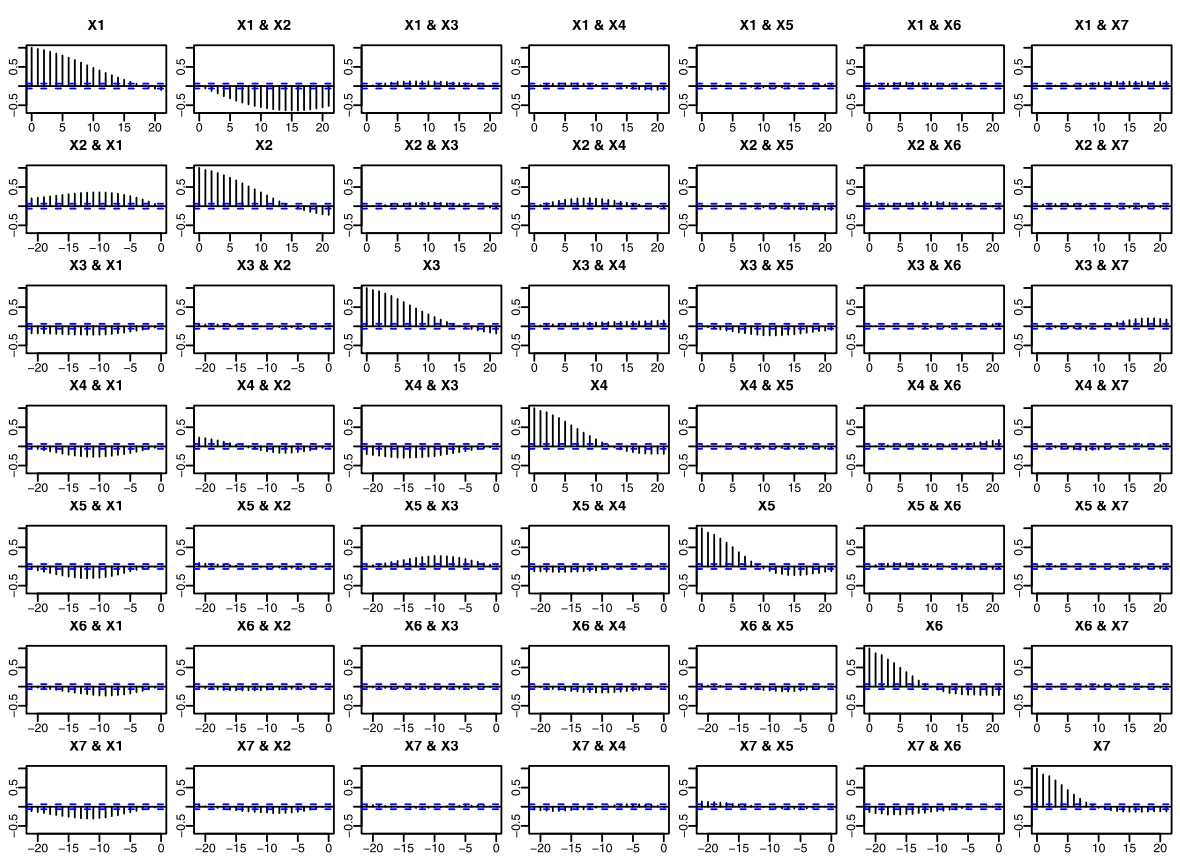

(b) Cross correlogram of the 7 transformed component time series

FIG. 2. Cross correlograms for Example 2. 


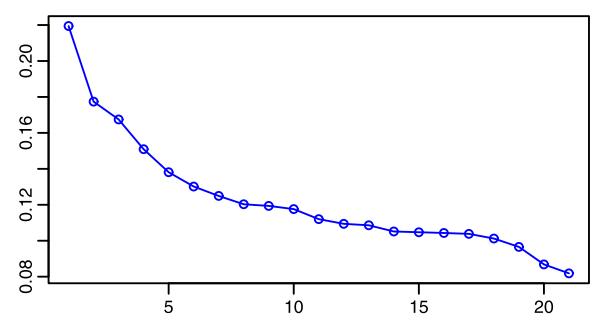

(a) Max cross correlations between pairs

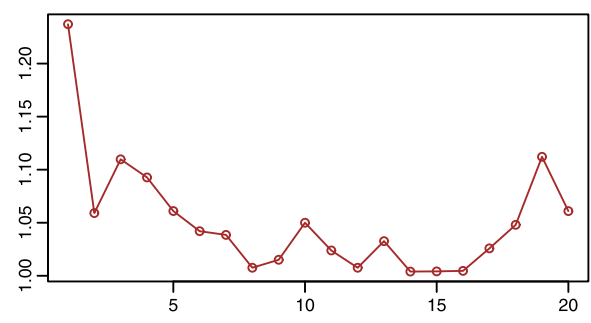

(b) Ratios of maximum cross correlations

FIG. 3. Example 2: (a) The maximum cross correlations, plotted in descending order, among each of the $\left(\begin{array}{l}7 \\ 2\end{array}\right)=21$ pairs component series of the transformed and prewhitened measles series. The maximization was taken over the lags between -20 to 20. (b) The ratios of two successive correlations in (a).

components, it has an adverse effect on forecasting, though it still outperforms the VAR and RVAR models. The alternative segmentation with the 3 groups takes into account more correlations, leading to the best forecasting performance in comparison with the other methods.

EXAMPLE 3. Now we consider the daily log-sales of a clothing brand in 25 provinces in China in 1 January 2008 - 9 December 2012 (i.e., $n=1805$ and $p=25$ ). All those series exhibit peaks before the Spring Festival (i.e., the Chinese New Year, typically around February). The cross correlogram of the 8 randomly selected component series in Figure 4 indicates the strong cross correlations over different time lags among the sales over different provinces. The strong periodic components with the period 7 indicate a regular sales pattern over 7 different weekdays. By applying the proposed segmentation transformation and the permutation based on the maximum cross correlations with $m=25$ in (2.11), the transformed 25 time series are divided into 24 group with only nonsingle-element group containing the 15th and the 16th transformed series. The same grouping is obtained

TABLE 2

Segmentations determined by different numbers of connected pairs for the transformed series in Example 2

\begin{tabular}{lcc}
\hline No. of connected pairs & No. of groups & Segmentation \\
\hline 1 & 6 & $\{4,5\},\{1\},\{2\},\{3\},\{6\},\{7\}$ \\
2 & 5 & $\{1,2\},\{4,5\},\{3\},\{6\},\{7\}$ \\
3 & 4 & $\{1,2,3\},\{4,5\},\{6\},\{7\}$ \\
4 & 3 & $\{1,2,3,7\},\{4,5\},\{6\}$ \\
5 & 2 & $\{1,2,3,6,7\},\{4,5\}$ \\
6 & 1 & $\{1, \ldots, 7\}$ \\
\hline
\end{tabular}




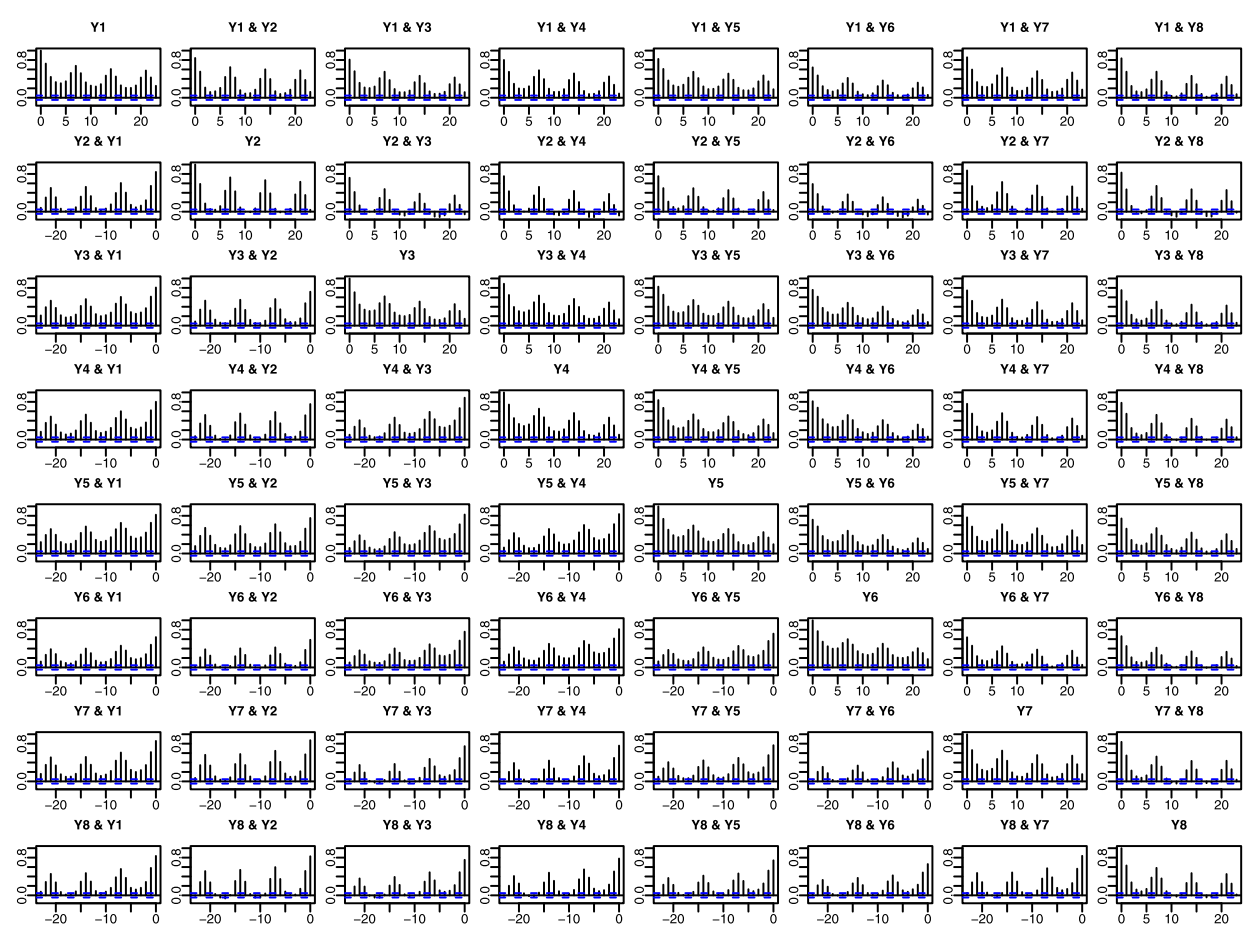

FIG. 4. Example 3: Cross correlogram of eight randomly selected log-sales series.

for $m$ between 14 and 30. Note for this example, we should not use small $m$ as the autocorrelations of the original data decay slowly; see Figure 4.

To compare the post-sample forecasting performance, we calculate one-stepahead and two-step-ahead forecasts for each of the daily log-sales in the last two weeks of the period. Table 1 lists the means and the standard deviations of the MSPEs across the 25 provinces. With $p=25$, the fitted VAR(2) model, selected by AIC, contain $2 \times 25 \times 25=1250$ parameters, leading to poor post-sample forecasting. The RVAR(2) model improves the forecasting a bit, but it is still significantly worse than the forecasting based on the approach of fitting a univariate AR model to each of the original series directly. Since the proposed segmentation leads to 24 subseries, it also fits univariate AR models to 23 (out of 25) transformed series, fits a 2-dimensional VAR model to the 15th and the 16th transformed series together. The proposed approach leads to much more accurate forecasts as both the mean and standard deviation are much smaller than those of the other three methods. The above comparison shows clearly that the cross correlations in the sales over different provinces are valuable information which can improve the forecasting for the future sales significantly. However, the endeavor to reduce the dimension by, for example, TS-PCA, is necessary in order to make use of this valuable information. We also consider an over-segmentation by regarding each component of the 
transformed series as an individual group, and an incomplete-segmentation with $\{5,15,16\}$ as a group and the other 22 components as 23 individual groups. Both of them show good performances.

EXAMPLE 4. The air pollution due to the fine particulate $\left(\mathrm{PM}_{2.5}\right)$ has aroused serious concerns in China. $\mathrm{PM}_{2.5}$ consists of airborne particles with aerodynamic diameters smaller than $2.5 \mu \mathrm{m}$. In this example, we consider the logarithmic daily average $\mathrm{PM}_{2.5}$ concentration readings at 84 monitoring stations in Beijing, Tianjin and Hebei in 1 January 2015-31 December 2016. Figure 5 is a map of those 84 stations. For this data set, $n=731$ and $p=84$. The readings at different locations are crossly correlated; see Figure 6 for the cross correlogram of six randomly selected stations.

Since the dimension $p$ is large, we choose $\widehat{\mathbf{S}}$ as in (3.5) with the threshold level $u$ determined by the method of Bickel and Levina (2008). The maximum cross correlation method in Section 2.2.2 divides the 84 transformed time series into 83 groups, with only one non-single-element group containing the 46th and the 83rd transformed series. In the post-sample forecasting for the daily readings in December 2016 (i.e., 31 days in total), we also include the over-segmentation with 84 groups (i.e., treating each transformed series as an individual group), and two incomplete segmentations with, respectively, 50 groups and 70 groups. The maximum group size is 8 for the segmentation with 50 groups, and is 4 for the segmentation with 70 groups. Those segmentations are obtained in the same manner

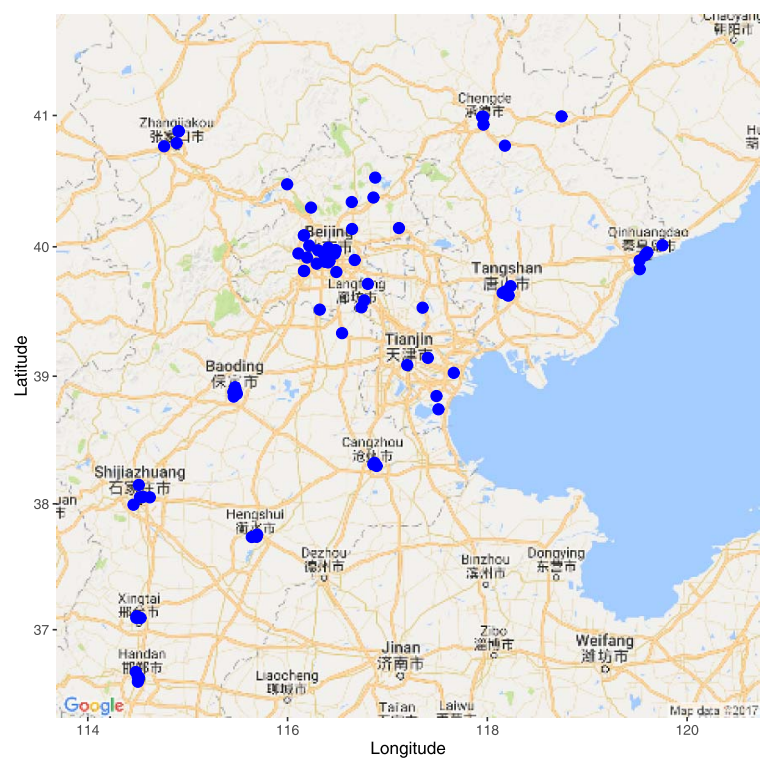

FIG. 5. Example 4: locations of $84 \mathrm{PM}_{2.5}$ monitoring stations. 

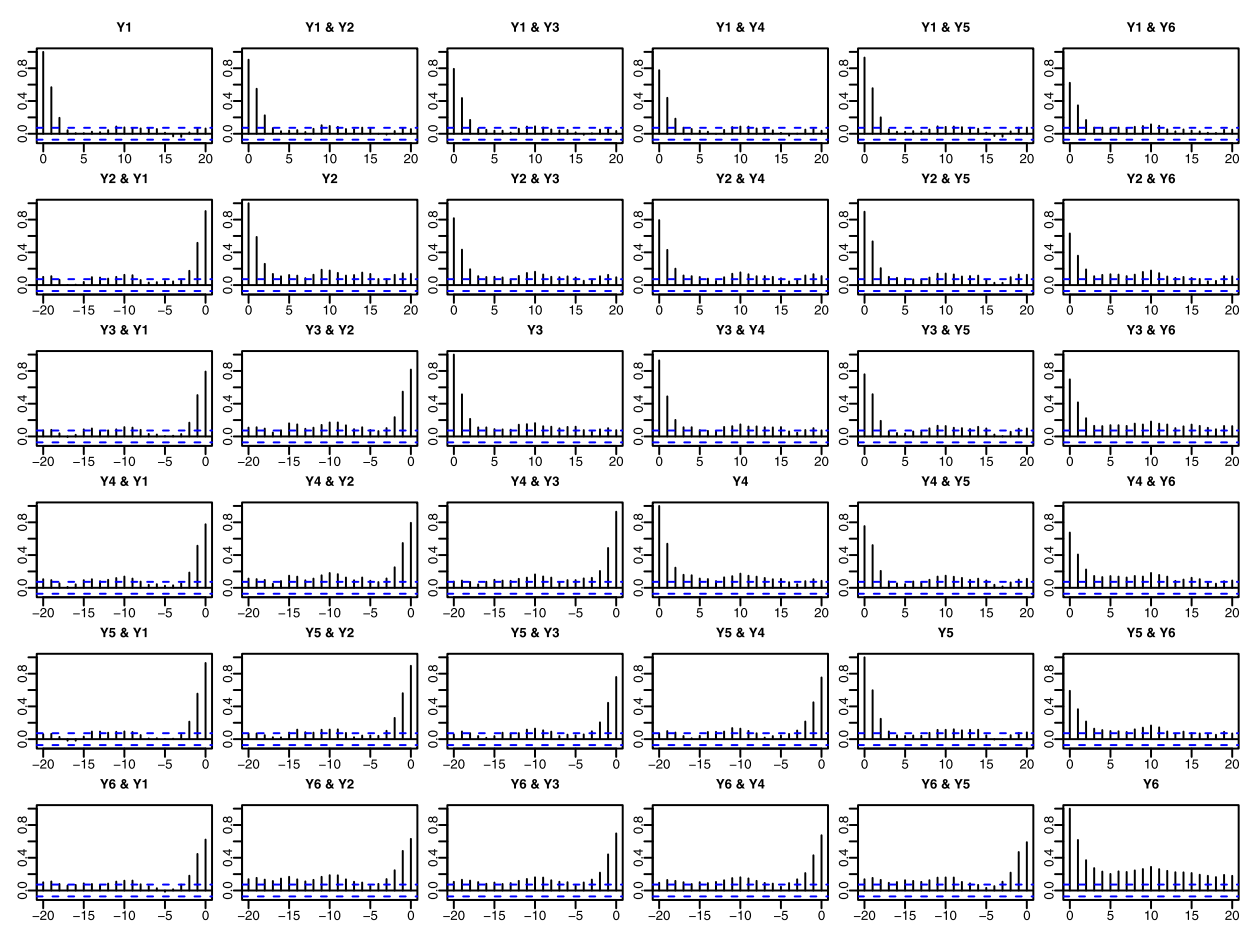

FIG. 6. Example 4: Cross correlogram of logarithmic daily $\mathrm{PM}_{2.5}$ readings at six randomly selected monitoring stations in Beijing, Tianjin and Hebei.

as in Example 2 (see also Table 2). With $p=84$, direct VAR is too crude to be attempted. Comparing to the univariate AR models for the original series, all the four segmentations provide more accurate one-step and two-step ahead predictions. It is worth pointing out that the difference due to using different segmentations is small.

5. Segmenting multivariate volatility processes. The methodology proposed in Section 2 can be readily extended to segment multivariate volatility processes. To this end, let $\mathbf{y}_{t}$ be a $p \times 1$ volatility process. Let $\mathcal{F}_{t}=\sigma\left(\mathbf{y}_{t}, \mathbf{y}_{t-1}, \ldots\right)$ and $\operatorname{Var}\left(\mathbf{y}_{t} \mid \mathcal{F}_{t-1}\right)=\boldsymbol{\Sigma}_{y}(t)$. Without loss of generality, we assume $\mathbb{E}\left(\mathbf{y}_{t} \mid \mathcal{F}_{t-1}\right)=\mathbf{0}$ and $\operatorname{Var}\left(\mathbf{y}_{t}\right)=\mathbf{I}_{p}$. Suppose that there exists an orthogonal matrix $\mathbf{A}$ for which $\mathbf{y}_{t}=\mathbf{A} \mathbf{x}_{t}$ and $\operatorname{Var}\left(\mathbf{x}_{t} \mid \mathcal{F}_{t-1}\right)=\operatorname{diag}\left\{\boldsymbol{\Sigma}_{1}(t), \ldots, \boldsymbol{\Sigma}_{q}(t)\right\}$ with $\boldsymbol{\Sigma}_{1}(t), \ldots, \boldsymbol{\Sigma}_{q}(t)$ being, respectively, $p_{1} \times p_{1}, \ldots, p_{q} \times p_{q}$ nonnegative definite matrices. Hence the latent $p$ dimensional volatility process $\mathbf{x}_{t}$ can be segmented into $q$ lower-dimensional processes, and there exist no conditional cross correlations across those $q$ processes.

Let $\mathbf{W}_{y}=\sum_{B \in \mathcal{B}_{t-1}}\left[\mathbb{E}\left\{\mathbf{y}_{t} \mathbf{y}_{t}^{\mathrm{T}} \mathbb{I}(B)\right\}\right]^{2}$ and $\mathbf{W}_{x}=\sum_{B \in \mathcal{B}_{t-1}}\left[\mathbb{E}\left\{\mathbf{x}_{t} \mathbf{x}_{t}^{\mathrm{T}} \mathbb{I}(B)\right\}\right]^{2}$, where $\mathcal{B}_{t-1}$ is a $\pi$-class and the $\sigma$-field generated by $\mathcal{B}_{t-1}$ equals to $\mathcal{F}_{t-1}$. Since it holds for any $B \in \mathcal{B}_{t-1}$ that $\mathbb{E}\left\{\mathbf{x}_{t} \mathbf{x}_{t}^{\mathrm{T}} \mathbb{I}(B)\right\}=\mathbb{E}\left\{\mathbb{I}(B) \mathbb{E}\left(\mathbf{x}_{t} \mathbf{x}_{t}^{\mathrm{T}} \mid \mathcal{F}_{t-1}\right)\right\}=\mathbb{E}\left[\mathbb{I}(B) \operatorname{diag}\left\{\boldsymbol{\Sigma}_{1}(t)\right.\right.$, 
$\left.\left.\ldots, \boldsymbol{\Sigma}_{q}(t)\right\}\right]$ is a block diagonal matrix, so is $\mathbf{W}_{x}$. Now (2.6) still holds for the newly defined $\mathbf{W}_{y}$ and $\mathbf{W}_{x}$. Thus $\mathbf{A}$ can be estimated exactly in the same manner as in Section 2.1. An estimator for $\mathbf{W}_{y}$ can be defined as $\widehat{\mathbf{W}}_{y}=$ $\sum_{B \in \mathcal{B}} \sum_{k=1}^{k_{0}}\left\{(n-k)^{-1} \sum_{t=k+1}^{n} \mathbf{y}_{t} \mathbf{y}_{t}^{\mathrm{T}} \mathbb{I}\left(\mathbf{y}_{t-k} \in B\right)\right\}^{2}$, where $\mathcal{B}$ is a set with elements $\left\{\mathbf{u} \in \mathbb{R}^{p}:\|\mathbf{u}\|_{2} \leq\left\|\mathbf{y}_{t}\right\|_{2}\right\}$ for $t=1, \ldots, n$; see Fan, Wang and Yao (2008). We illustrate this idea by a real data example.

EXAMPLE 5. We consider the daily returns of the stocks of Walt Disney Company, Wells Fargo \& Company, Honeywell International Inc., MetLife Inc., H \& R Block Inc. and Cognizant Technology Solutions Corporation in 14 July 2008-11 July 2014. For this data set, $n=1509$ and $p=6$. Denote by $\mathbf{y}_{t}=\left(y_{1, t}, \ldots, y_{6, t}\right)^{\mathrm{T}}$ the returns on the $t$ th day. By fitting each return series a $\operatorname{GARCH}(1,1)$ model, we calculate the residuals $\varepsilon_{i, t}=y_{i, t} / \widehat{\sigma}_{i, t}$ for $i=1, \ldots, 6$, where $\widehat{\sigma}_{i, t}$ denotes the predicted volatility for the $i$ th return at time $t$ based on the fitted $\operatorname{GARCH}(1,1)$ model. The cross correlogram of the residual series are plotted in Figure 7(a), which shows the strong and significant concurrent correlations across all residual series. It indicates clearly that $\operatorname{Var}\left(\mathbf{y}_{t} \mid \mathcal{F}_{t-1}\right)$ is not a block diagonal matrix. We also apply the traditional PCA to the 6 returns series, the cross correlogram of prewhitened series is shown in Figure 7(b). There are also strong and significant concurrent correlations across the residual series; see Panels $(1,2),(2,3),(3,4),(2,5)$ and $(6,4)$. This indicates all the principal components should not be modelled separately. Now we apply the segmentation transform stated above. We repeat the whitening process above for the transformed series $\widehat{\mathbf{x}}_{t}$, that is, fit a $\operatorname{GARCH}(1,1)$ model for each of the component series of $\widehat{\mathbf{x}}_{t}$ and calculate the residuals. Figure 8 presents the cross correlogram of these new residual series. There exist almost no significant cross correlations among the residual series. This is the significant evidence to support the assertion that $\operatorname{Var}\left(\mathbf{x}_{t} \mid \mathcal{F}_{t-1}\right)$ is a diagonal matrix. For this example, the segmentation method leads to the conditional uncorrelated components of Fan, Wang and Yao (2008).

6. Final remarks. This paper proposes a contemporaneous linear transformation to segment a multiple time series into several both contemporaneously and serially uncorrelated subseries. The method is simple, and can be used as a preliminary step to reduce a high-dimensional time series modelling problem into several lower-dimensional problems. The reduction of dimensionality is often substantial and effective.

The method is abbreviated as TS-PCA, as it can be viewed as a version of PCA for multiple time series. Like the standard PCA, TS-PCA technically also boils down to an eigenanalysis for a positive definite matrix. The difference is that the intended segmentation is not guaranteed to exist. However, one of the strengths of the proposed TS-PCA is that even when the segmentation assumption is invalid, it provides some approximate segmentations which ignore some minor (though still 


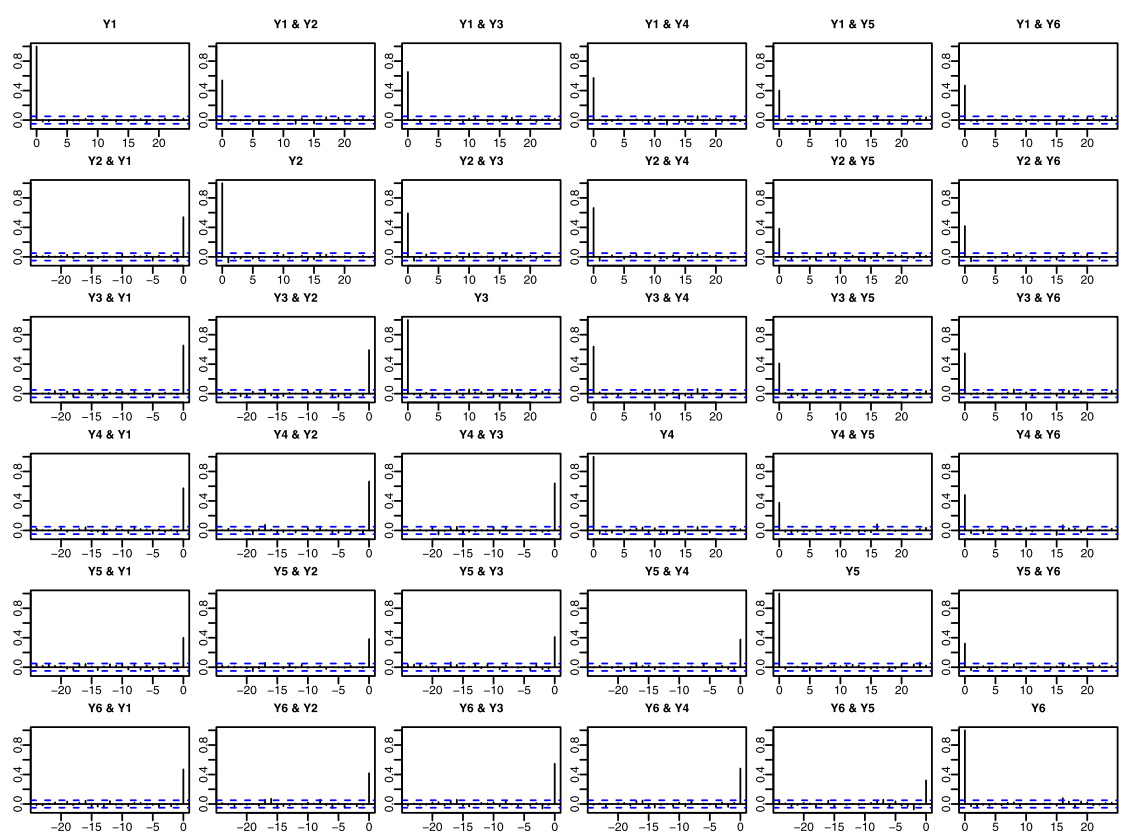

(a) Cross correlogram of the residuals resulted from fitting each original component series a $\operatorname{GARCH}(1,1)$ model

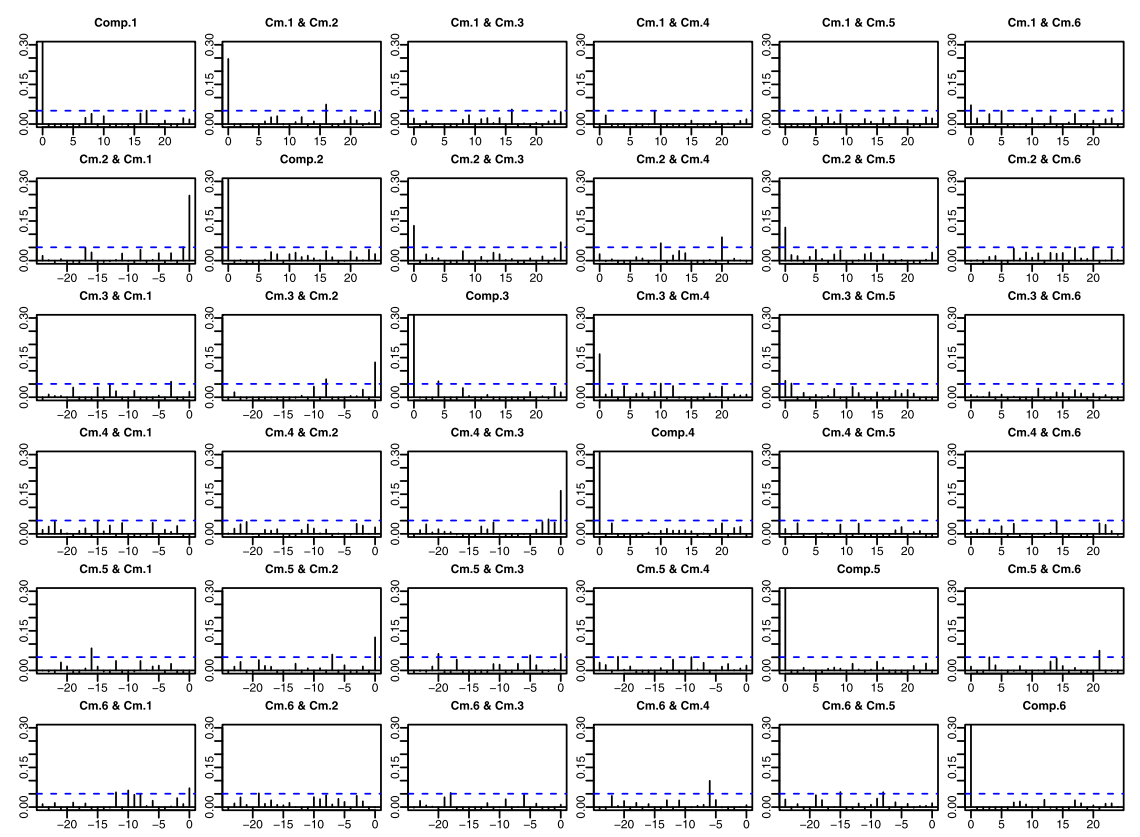

(b) Cross correlogram of the residuals resulted from fitting each series of PCA components a $\operatorname{GARCH}(1,1)$ model

FIG. 7. Cross correlograms for Example 5. 

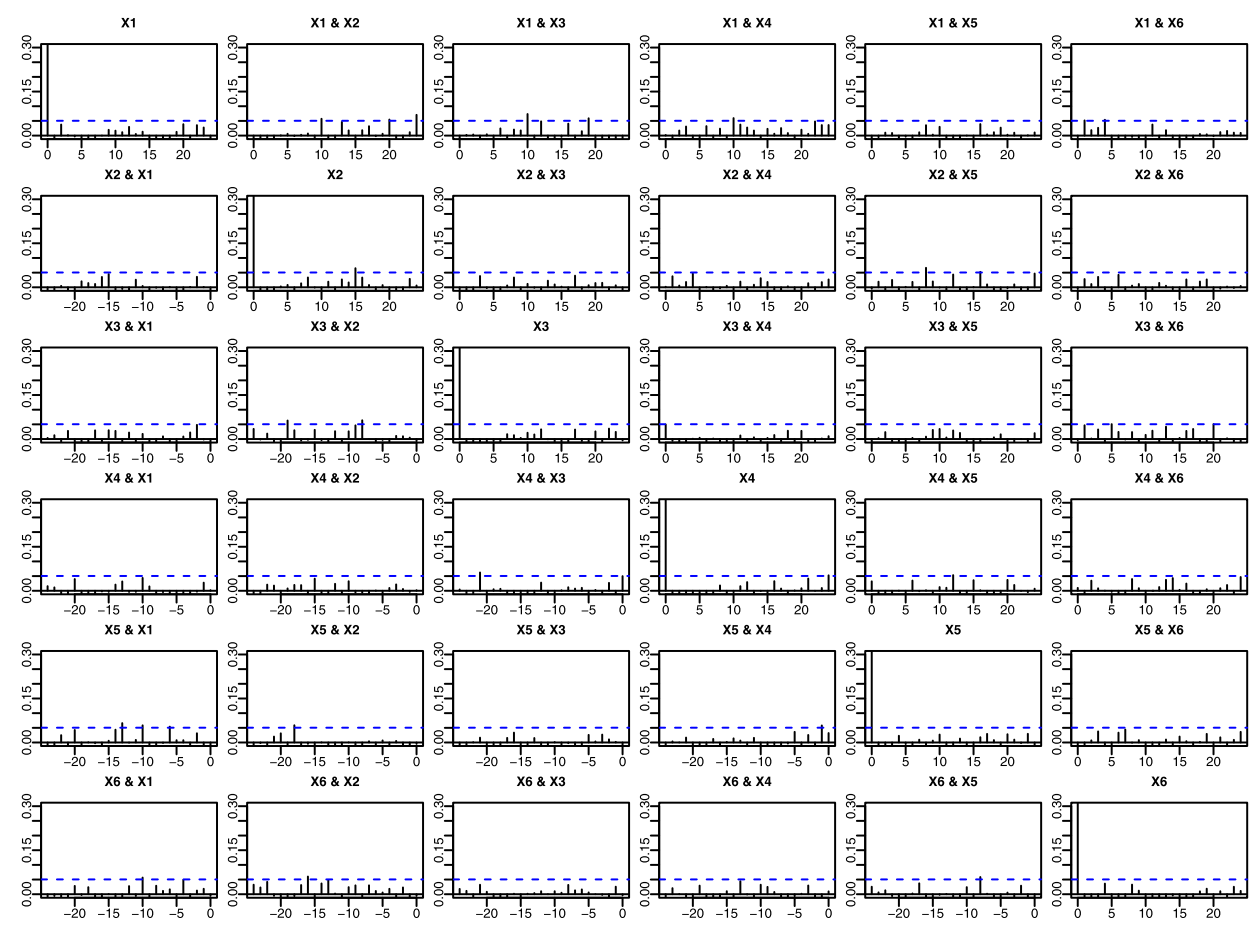

FIG. 8. Cross correlogram of the residuals resulted from fitting each component series of the transformed series $\widehat{\mathbf{x}}_{t}$ with a $\operatorname{GARCH}(1,1)$ model in Example 5.

significant) cross correlations, and thus, lead to parsimonious modelling strategies. Those parsimonious strategies often bring in improvements in, for example, forecasting future values; see, for example, Example 2. Furthermore, when the dimension of time series is large, TS-PCA is necessary in order to utilize the information across different component series effectively; see, for example, Examples 3 and 4.

We have conducted some post-sample forecasting comparison with several real data sets including some not reported in the paper. The forecasting based on the proposed TS-PCA always outperforms that for the original data. We give one explanation as follows. It follows from (2.6) that $\Omega \equiv \operatorname{tr}\left(\mathbf{W}_{y}\right)-p=$ $\sum_{k=1}^{k_{0}} \sum_{i, j=1}^{p} \rho_{y, i j}^{2}(k)=\operatorname{tr}\left(\mathbf{W}_{x}\right)-p=\sum_{k=1}^{k_{0}} \sum_{i, j=1}^{p} \rho_{x, i j}^{2}(k)$, where $\rho_{y, i j}(k)$ and $\rho_{x, i j}(k)$ denote, respectively, the cross correlation at lag $k$ between the $i$ th and the $j$ th components of $\mathbf{y}_{t}$ and $\mathbf{x}_{t}$. Since the future prediction is based on the serial correlations, $\Omega$ can be taken as a measure for the predictive strength, which is the same for $\mathbf{y}_{t}$ and $\mathbf{x}_{t}$. To make use of the full predictive strength of $\mathbf{y}_{t}$, we need to model the $p$-vector process appropriately to catch all the autocorrelations and cross correlations (over different time lags) among the $p$ components of $\mathbf{y}_{t}$. In contrast, such a task for $\mathbf{x}_{t}$ is much easier as it can be divided into $q$ lower-dimensional problems. In the ideal situation when $q=p$, that is, $\rho_{x, i j}(k)=0$ for any $i \neq j$, we 
just need to model all the component series of $\mathbf{x}_{t}$ separately in order to make the full use of the overall predictive strength.

Acknowledgements. The authors sincerely thank the Co-Editor, Associate Editor and three referees for their constructive suggestions and comments that led to substantial improvement of the paper.

\section{SUPPLEMENTARY MATERIAL}

Supplement to "Principal component analysis for second-order stationary vector time series" (DOI: 10.1214/17-AOS1613SUPP; .pdf). This supplement contains simulation studies and all technical proofs.

\section{REFERENCES}

ANDERSON, T. W. (1963). The use of factor analysis in the statistical analysis of multiple time series. Psychometrika 28 1-25. MR0165648

BACK, A. D. and WeIGEND, A. S. (1997). A first application of independent component analysis to extracting structure from stock returns. Int. J. Neural Syst. 8 473-484.

BAI, J. and NG, S. (2002). Determining the number of factors in approximate factor models. Econometrica 70 191-221. MR1926259

Belouchrani, A., Abed-Meraim, K., Cardoso, J.-F. and Moulines, E. (1997). A blind source separation technique using second-order statistics. IEEE Trans. Signal Process. 45434 444.

Bickel, P. J. and Levina, E. (2008). Covariance regularization by thresholding. Ann. Statist. 36 2577-2604. MR2485008

Box, G. E. P. and Jenkins, G. M. (1970). Times Series Analysis. Forecasting and Control. HoldenDay, San Francisco, CA-London-Amsterdam. MR0272138

Box, G. E. P. and TIAO, G. C. (1977). A canonical analysis of multiple time series. Biometrika 64 355-365. MR0519089

Brillinger, D. R. (1975). Time Series: Data Analysis and Theory. Holt, Rinehart and Winston, Inc., New York-Montreal, QC-London. MR0443257

BRockWell, P. J. and DAVIS, R. A. (1996). Introduction to Time Series and Forecasting. Springer, New York. MR1416563

CARdoso, J. (1998). Multidimensional independent component analysis. In Proceedings of the 1998 IEEE Int. Conf. Acoustics, Speech and Signal Processing 4 1941-1944.

ChAnG, J., GuO, B. and YAO, Q. (2015). High dimensional stochastic regression with latent factors, endogeneity and nonlinearity. J. Econometrics 189 297-312. MR3414901

Chang, J., Guo, B. and YAO, Q. (2018). Supplement to "Principal component analysis for secondorder stationary vector time series." DOI: 10.1214/17-AOS1613SUPP.

CHANG, J., YAO, Q. and ZHOU, W. (2017). Testing for high-dimensional white noise using maximum cross-correlations. Biometrika 104 111-127. MR3626482

DAVIS, R. A., ZANG, P. and ZHENG, T. (2016). Sparse vector autoregressive modeling. J. Comput. Graph. Statist. 25 1077-1096. MR3572029

FAN, J., WANG, M. and YAO, Q. (2008). Modelling multivariate volatilities via conditionally uncorrelated components. J. R. Stat. Soc. Ser. B. Stat. Methodol. 70 679-702. MR2523899

FAN, J. and YAO, Q. (2003). Nonlinear Time Series: Nonparametric and Parametric Methods. Springer, New York. MR1964455 
Forni, M., Hallin, M., LipPI, M. and Reichlin, L. (2005). The generalized dynamic factor model: One-sided estimation and forecasting. J. Amer. Statist. Assoc. 100 830-840. MR2201012

GUO, S., WANG, Y. and YAO, Q. (2016). High-dimensional and banded vector autoregressions. Biometrika 103 889-903. MR3620446

HAN, F., LU, H. and LIU, H. (2015). A direct estimation of high dimensional stationary vector autoregressions. J. Mach. Learn. Res. 16 3115-3150. MR3450535

HUANG, D. and TSAY, R. S. (2014). A refined scalar component approach to multivariate time series modeling. Manuscript.

Hyvärinen, A., Karhunen, J. and OJA, E. (2001). Independent Component Analysis. Wiley, New York.

Jakeman, A. J., Steele, L. P. and Young, P. C. (1980). Instrumental variable algorithms for multiple input systems described by multiple transfer functions. IEEE Trans. Syst. Man Cybern. Syst. 10 593-602.

LAM, C. and YAO, Q. (2012). Factor modeling for high-dimensional time series: Inference for the number of factors. Ann. Statist. 40 694-726. MR2933663

LAM, C., YAO, Q. and BATHIA, N. (2011). Estimation of latent factors for high-dimensional time series. Biometrika 98 901-918. MR2860332

LEDOIT, O. and WoLF, M. (2004). A well-conditioned estimator for large-dimensional covariance matrices. J. Multivariate Anal. 88 365-411. MR2026339

LIU, W., XIAO, H. and WU, W. B. (2013). Probability and moment inequalities under dependence. Statist. Sinica 23 1257-1272. MR3114713

LÜtKepohl, H. (2005). New Introduction to Multiple Time Series Analysis. Springer, Berlin. MR2172368

MATTESON, D. S. and TSAY, R. S. (2011). Dynamic orthogonal components for multivariate time series. J. Amer. Statist. Assoc. 106 1450-1463. MR2896848

PAN, J. and YAO, Q. (2008). Modelling multiple time series via common factors. Biometrika 95 365-379. MR2521589

PAPARoditis, E. and Politis, D. N. (2012). Nonlinear spectral density estimation: Thresholding the correlogram. J. Time Series Anal. 33 386-397. MR2915091

PEÑA, D. and Box, G. E. P. (1987). Identifying a simplifying structure in time series. J. Amer. Statist. Assoc. 82 836-843. MR0909990

ReInsel, G. C. (1993). Elements of Multivariate Time Series Analysis. Springer, New York. MR1238940

Rio, E. (2000). Théorie Asymptotique des Processus Aléatoires Faiblement Dépendants. Mathématiques \& Applications (Berlin) [Mathematics \& Applications] 31. Springer, Berlin. MR2117923

SARKAR, S. K. and CHANG, C.-K. (1997). The Simes method for multiple hypothesis testing with positively dependent test statistics. J. Amer. Statist. Assoc. 92 1601-1608. MR1615269

ShojaIE, A. and Michailidis, G. (2010). Discovering graphical Granger causality using the truncated lasso penalty. Bioinformatics 26 517-523.

SimES, R. J. (1986). An improved Bonferroni procedure for multiple tests of significance. Biometrika 73 751-754. MR0897872

SONG, S. and BICKEL, P. J. (2011). Large vector auto regressions. Available at arXiv:1106.3519.

Stewart, G. W. and Sun, J. G. (1990). Matrix Perturbation Theory. Academic Press, Boston, MA. MR1061154

Stock, J. H. and WATSON, M. W. (2002). Forecasting using principal components from a large number of predictors. J. Amer. Statist. Assoc. 97 1167-1179. MR1951271

STOCK, J. H. and WATSON, M. W. (2005). Implications of dynamic factor models for VAR analysis. Available at: www.nber.org/papers/w11467.

Theis, F. J., Meyer-BAese, A. and LANG, E. W. (2004). Second-order blind source separation based on multi-dimensional autocovariances. In Independent Component Analysis and Blind Signal Separation (C. G. Puntonet and A. Prieto, eds.) 726-733. Springer, Berlin. 
TiaO, G. C. and Tsay, R. S. (1989). Model specification in multivariate time series. J. Roy. Statist. Soc. Ser. B 51 157-213. With discussion. MR1007452

TONG, L., XU, G. and KaILATH, T. (1994). Blind identification and equalization based on secondorder statistics: A time domain approach. IEEE Trans. Inform. Theory 40 340-349.

TSAY, R. S. (2014). Multivariate Time Series Analysis: With R and Financial Applications. Wiley, Hoboken, NJ. MR3236787

J. CHANG

B. GUO

SCHOOL OF STATISTICS

AND

Center of Statistical Research

SOUTHWESTERN UNIVERSITY OF FINANCE AND ECONOMICS

Chengdu, Sichuan 611130

CHINA

E-MAIL: changjinyuan@swufe.edu.cn guobin@swufe.edu.cn
Q. YAO

DEPARTMENT OF STATISTICS

LONDON SCHOOL OF ECONOMICS AND POLITICAL SCIENCE

LONDON, WC2A 2AE

UNITED KINGDOM

E-MAIL: q.yao@1se.ac.uk 